\title{
Achievable Rate Region of the Zero-Forcing Precoder in a $2 \times 2$ MU-MISO Broadcast VLC Channel with Per-LED Peak Power Constraint and Dimming Control
}

\author{
Amit Agarwal and Saif Khan Mohammed
}

\begin{abstract}
In this paper, we consider the $2 \times 2$ multi-user multiple-input-single-output (MU-MISO) broadcast visible light communication (VLC) channel with two light emitting diodes (LEDs) at the transmitter and a single photo diode (PD) at each of the two users. We propose an achievable rate region of the Zero-Forcing (ZF) precoder in this $2 \times 2$ MU-MISO VLC channel under a per-LED peak and average power constraint, where the average optical power emitted from each LED is fixed for constant lighting, but is controllable (referred to as dimming control in IEEE 802.15.7 standard on VLC). We analytically characterize the proposed rate region boundary and show that it is Pareto-optimal. Further analysis reveals that the largest rate region is achieved when the fixed per-LED average optical power is half of the allowed per-LED peak optical power. We also propose a novel transceiver architecture where the channel encoder and dimming control are separated which greatly simplifies the complexity of the transceiver. A case study of an indoor VLC channel with the proposed transceiver reveals that the achievable information rates are sensitive to the placement of the LEDs and the PDs. An interesting observation is that for a given placement of LEDs in a $5 \mathrm{~m} \times 5 \mathrm{~m} \times$ $3 \mathrm{~m}$ room, even with a substantial displacement of the users from their optimum placement, reduction in the achievable rates is not significant. This observation could therefore be used to define "coverage zones" within a room where the reduction in the information rates to the two users is within an acceptable tolerance limit.
\end{abstract}

Index Terms - Visible light communication, rate region, zeroforcing, multi-user, multiple-input-multiple-output.

\section{INTRODUCTION}

Visible light communication (VLC) is a form of optical wireless communication (OWC) technology which can provide high speed indoor wireless data transmission using existing infrastructure for lighting. One distinctive advantage of VLC technology is that it utilizes the unused visible band of the electromagnetic spectrum and does not interfere with the existing radio frequency (RF) communication in the UHF (Ultra High Frequency) band [1], [2].

In VLC systems, it is common to use intensity modulation (IM) via light emitting diode (LEDs) for transmission of information signal and direct detection (DD) via photodiodes (PDs)

The authors are with the Department of Electrical Engineering, Indian Institute of Technology Delhi (IITD), New delhi, India. Saif Khan Mohammed is also associated with Bharti School of Telecommunication Technology and Management (BSTTM), IIT Delhi. Email: saifkmohammed@gmail.com. This work is supported by the Visvesvaraya Young Faculty Research Fellowship (YFRF) of the Ministry of Electronics and Information Technology, Govt. of India. for the recovery of the information signal [1]. Contrary to RF systems, in VLC systems the modulation symbols must be non-negative and real valued as information is communicated by modulating the power/intensity of the light emitted by the optical source (LED). The modulation symbols are also constrained to be less than a pre-determined value as the intensity of the light emitted by the LED is peak constrained due to safety regulations and also due to the limited linear range of the transfer function of LEDs [1], [3]. Moreover, due to constant lighting the mean value of the modulation symbol is also fixed (i.e., non-time varying) and can be adjusted according to the users' requirement (dimming target) [4], [5].

Due to these constraints, analysis performed for RF systems is not directly applicable to VLC systems. For example, the capacity of the RF single-input-single-output (SISO) additive white Gaussian noise (AWGN) channel is well known and it has been shown that the Gaussian input distribution is capacity achieving. For the case of the optical wireless AWGN SISO channel with IM/DD transceiver, closed form expression for the capacity is still not known, though several inner and outer bounds have been proposed [6]-[8]. However, it has been shown that the capacity achieving input distribution for the IM/DD SISO AWGN optical wireless channel is discrete [9], and has been computed numerically in [10]. Similarly, for the case of dimmable VLC IM/DD SISO channel with peak constraint, there is no closed from expression for the capacity. However following a similar approach as in [6], an upper and lower bound is presented in [11].

Recently, there has been a lot of interest in multiuser multiple-input multiple-output/single-output (MUMIMO/MISO) VLC systems, where multiple LEDs are used for information transmission to multiple non-cooperative PDs (users) [12], [1]. Such systems have been shown to enhance the system sum rate when compared to SISO VLC systems [13], [14] .

In [13], the information sum rate of MU-MIMO VLC broadcast systems has been studied under the non-negativity constraint on the signal transmitted from each LED, and also a per-LED average transmitted power constraint with no dimming control. The block diagonalization precoder in [13] is used to suppress the multi-user interference and the numerically computed achievable sum rate is shown to be sensitive to the placement of the users and the rotation of the PDs. However, they do not consider peak power constraints 
which is important due to eye safety regulations and also due to the requirement of limited interference to other VLC systems.

Per-LED peak and average power constraint has been considered in [14], where the sum-rate of the zero forcing (ZF) precoder is maximized in a IM/DD based MU-MIMO/MISO VLC systems. However, in many practical scenarios fairness is required and therefore maximizing the sum rate might not always be the desired operating regime. For example we would like to find the maximum possible rate such that each user gets the same rate. Such operating points can only be obtained from the rate region characterization of the MU-MIMO VLC systems. In [15], authors have proposed inner and outer bounds on the capacity region of a two user IM/DD broadcast VLC system where the transmitter has a single LED and each user has a single PD. Per-LED average and peak power constraints are considered. The authors have extended their work to more than two users in [16]. However, in both [15] and [16], the transmitter has only one LED. Furthermore, dimming control is not considered in [13]-[16].

The capacity/achievable rate region of a IM/DD based VLC broadcast channel where the transmitter has $N>1$ LEDs and $M>1$ users having one PD each, is still an open and challenging problem, primarily due to the non-negativity, peak and average constraints on the electrical signal input to each LED.

In this paper, we consider the smallest instance of this open problem along with dimming control, i.e., with $N=2$ LEDs at the transmitter and $M=2$ users (each having one PD). Dimming control is required in indoor VLC systems since the illumination should not vary with time on its own and should be controllable by the users. Therefore, in this paper, in addition to the peak and non-negativity constraints, we constrain the average optical power radiated by each LED to be fixed, i.e., non-time varying. Subsequently in this paper we refer to this system as the $2 \times 2$ MU-MISO VLC broadcast system.

The major contributions of this paper are as follows:

1) In Section III, we propose an achievable rate region for the $2 \times 2$ MU-MISO VLC broadcast system with the $\mathrm{ZF}$ precoder. In this section through analysis we show that the per-LED non-negativity and peak constraint restricts the information symbol vector for the two users (i.e., $\left.\left(u_{1}, u_{2}\right)\right)$ to lie within a parallelogram $R_{/ /}$. Each achievable rate pair $\left(R_{1}, R_{2}\right)$ then corresponds to a rectangle which lies within $R_{/ /}$. The rate $R_{i}, i=1,2$ to the $i^{t h}$ user depends on the length of the rectangle along the $u_{i}$-axis. Due to the same average optical power constraint at each LED, these rectangles should also have their midpoint (i.e., point of intersection of the diagonals of the rectangle) at a fixed point on the diagonal of $R_{/ /}$denoted by D. ${ }^{1}$ This fixed point $\mathrm{D}$ on the diagonal of $R_{/ /}$is non-time varying, but can be controlled by the user depending upon the illumination requirement. This feature of the proposed system enables

\footnotetext{
${ }^{1}$ Out of the two diagonals of $R_{/ /}$, we refer to the one which has one end point at the origin $\left(u_{1}, u_{2}\right)=(0,0)$.
}

dimming control.

2) In Section III, We also mathematically define the proposed rate region of the $\mathrm{ZF}$ precoder for a fix dimming target.

3) In Section IV, we analytically characterize the boundary of the proposed rate region by deriving explicit expressions for the largest possible length along the $u_{2}$-axis of some rectangle inside $R_{/ /}$whose midpoint coincides with the fixed point $\mathrm{D}$ on the diagonal of $R_{/ /}$ and whose length along the $u_{1}$-axis is given. Through analysis we also show that the rate region boundary is Pareto-optimal.

4) We also analyze the variation in the rate region with change in the dimming level. In depth analysis reveals that the largest rate region is achieved when the fixed point $\mathrm{D}$ lies at the midpoint of the diagonal of $R_{/ /}$, i.e., when the fixed per-LED average optical transmit power is half of the per-LED peak optical power.

5) For practical scenarios with fairness constraints, through analysis we show that the largest achievable rate pair $\left(R_{1}, R_{2}\right)$ such that $R_{2}=\alpha R_{1}$ is given by the unique intersection of the proposed rate region boundary with the straight line $R_{2}=\alpha R_{1}$.

6) In Section $V$, from the point of view of practical implementation we also propose a novel transceiver architecture where the same channel encoder can be used irrespective of the level of dimming control.

7) Analytical results have been supported with numerical simulations in Section VI. It is observed that for a fixed placement of the two LEDs, the achievable information rates are a function of the placement of the two PDs/users. Specifically, we observe that for a given placement of the two LEDs, there exists an optimal placement of the two users which maximizes the symmetric rate. Another interesting observation is that in a $5 \mathrm{~m} \times 5 \mathrm{~m} \times 3 \mathrm{~m}$ (height) room with the two LEDs attached to the ceiling and the two PDs placed in the horizontal plane at a height of $50 \mathrm{~cm}$ above the floor, even a user displacement of $60 \mathrm{~cm}$ from the optimal placement results in only approx. a 10 percent reduction in the symmetric rate when compared to the symmetric rate with the optimal placement of $\mathrm{PDs}^{2}$. This allows for substantial mobility of the user terminals around their optimal placement which is specially desirable when the user terminals are mobile/portable. A practical application of the results derived in this paper could be in defining coverage zones for the PDs/users, i.e., the maximum allowable displacement of the users for a fixed desired upper limit on the percentage loss in the achievable information rates.

\section{SySTEM MODEL}

We consider a $2 \times 2 \mathrm{IM} / \mathrm{DD}$ MU-MISO VLC broadcast system. The transmitter of the MISO system is equipped with two LEDs and each user has a single photo-diode

\footnotetext{
${ }^{2}$ For this study the dimming control is such that the average optical power radiated from each LED is 30 percent of the peak allowed optical power
} 


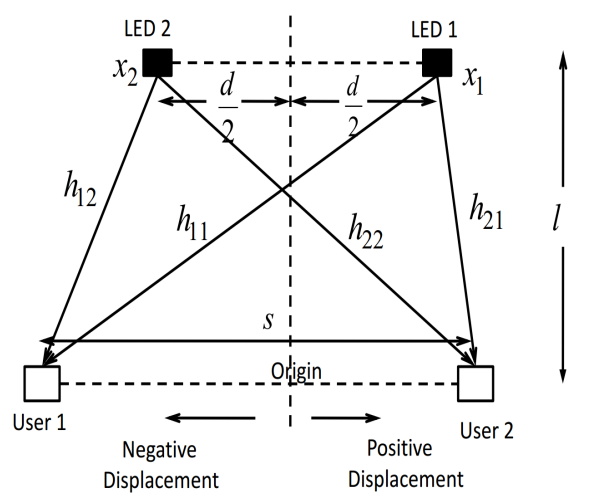

Fig. 1: $2 \times 2$ IM/DD MU-MISO VLC broadcast system.

(PD) (see Fig. 1). ${ }^{3}$ The LED converts the information carrying electrical signal to an intensity modulated optical signal and the PD at each user converts the received optical signal to electrical signal. The transmitter performs beamforming of the information symbols towards the two non-cooperative users. Let $u_{1} \in \mathcal{U}_{1}$ and $u_{2} \in \mathcal{U}_{2}$ be the information symbols intended for the first and second user respectively, where $\mathcal{U} 1$ and $\mathcal{U}_{2}$ are the information symbol alphabets for user 1 and user 2 respectively. Let $x_{i}$ be the optical power transmitted from the $i^{\text {th }} \operatorname{LED}(i=1,2)$. At any time instance, the transmitted optical power vector $\boldsymbol{x} \triangleq\left[\begin{array}{ll}x_{1} & x_{2}\end{array}\right]^{T}$ is given by

$$
\boldsymbol{x}=\boldsymbol{A u},
$$

where $\boldsymbol{u} \triangleq\left[\begin{array}{ll}u_{1} & u_{2}\end{array}\right]^{T}$ and $\boldsymbol{A} \in \mathbb{R}^{2 \times 2}$ is the beamforming matrix. In this paper, we consider the following power constraints for our dimmable VLC system.

The instantaneous power transmitted from each LED is non negative and is less than some maximum limit $P_{0}$ due to skin and eye safety regulations [3]. Further, such a maximum limit on the transmitted power is required also due to limited interference requirement to the neighboring VLC systems, i.e.

$$
0 \leq x_{i} \leq P_{0}, i=1,2 \text {. }
$$

Since our VLC system is dimmable we further impose a per-LED average power constraint of the type

$$
E\left[x_{i}\right]=\xi P_{0}, i=1,2,
$$

where $0 \leq \xi \leq 1$ is the dimming target [5]. For the sake of analysis, we define $x_{i}^{\prime} \triangleq \frac{x_{i}}{P_{0}}, i=1,2$ as the normalized power transmitted from each LED. Consequently, the normalized optical power transmitted from each LED must satisfy the following constraints given by

$$
0 \leq x_{i}^{\prime} \leq 1 \quad \& \quad E\left[x_{i}^{\prime}\right]=\xi, i=1,2 .
$$

Assuming $y_{k}, k=1,2$ to be the normalized received electrical signal at the $k^{\text {th }}$ user (after scaling down by $P_{0}$ ), the

\footnotetext{
${ }^{3}$ Since each of the two users has a single PD we will be interchangeably using user and PD in subsequent discussions.
}

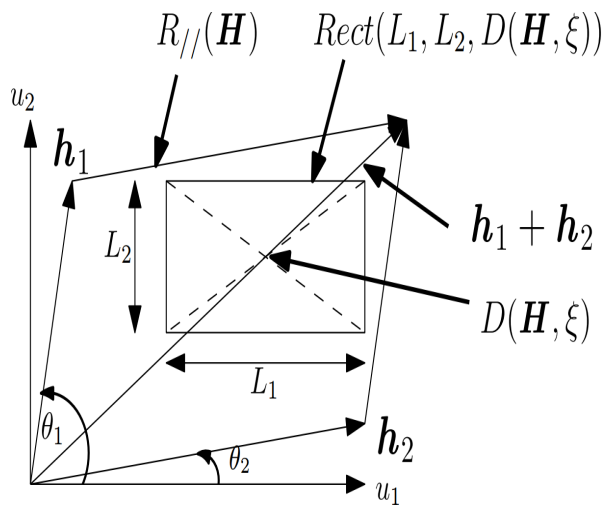

Fig. 2: The information vector $\boldsymbol{u}$ is constrained to lie within the parallelogram $R_{/ /}(\boldsymbol{H})$ whose non-parallel sides are $\boldsymbol{h}_{1}$ and $\boldsymbol{h}_{2}$. The rectangular region $\mathcal{U}_{1} \times \mathcal{U}_{2}$ whose length along the $u_{1}$ axis is $L_{1}$ and that along the $u_{2}$ axis is $L_{2}$ and whose center lies at $D(\boldsymbol{H}, \xi)$ is denoted by $\operatorname{Rect}\left(L_{1}, L_{2}, D(\boldsymbol{H}, \xi)\right)$.

normalized received signal vector is given by ${ }^{4}$

$$
\begin{array}{ll} 
& \underbrace{\left[\begin{array}{l}
y_{1} \\
y_{2}
\end{array}\right]}_{\triangleq \boldsymbol{y}}=\underbrace{\left[\begin{array}{ll}
h_{11} & h_{12} \\
h_{21} & h_{22}
\end{array}\right]}_{\triangleq \boldsymbol{H}} \underbrace{\left[\begin{array}{l}
x_{1}^{\prime} \\
x_{2}^{\prime}
\end{array}\right]}_{\triangleq \boldsymbol{x}^{\prime}}+\underbrace{\left[\begin{array}{l}
n_{1} \\
n_{2}
\end{array}\right]}_{\triangleq \boldsymbol{n}}, \\
\text { s.t. } & 0 \leq x_{i}^{\prime} \leq 1, \quad E\left[x_{i}^{\prime}\right]=\xi, \quad i=1,2 .
\end{array}
$$

where $\boldsymbol{H} \triangleq\left[h_{k i}\right]_{2 \times 2}$ is the channel gain matrix. The channel gain coefficients between the $i^{\text {th }}$ LED and the $k^{\text {th }}$ user is denoted by $h_{k i}, i=1,2, k=1,2 .{ }^{5}$ We further define $\boldsymbol{h}_{1} \triangleq$ $\left[\begin{array}{ll}h_{11} & h_{21}\end{array}\right]^{T}$ and $\boldsymbol{h}_{2} \triangleq\left[\begin{array}{ll}h_{12} & h_{22}\end{array}\right]^{T}$ to be the channel vectors from LED 1 and LED 2 respectively. Further, $n_{1}$ and $n_{2}$ are the sum of the thermal noise and ambient light-induced shot noise at the respective users ${ }^{6}$ and are independent of $x_{1}^{\prime}$ and $x_{2}^{\prime}$ [12]. The noise signals are i.i.d. zero mean real AWGN with variance $\sigma^{2} / P_{0}^{2}$, where $\sigma^{2}$ is the variance of the noise before the scaling down of the received signal by $P_{0}$, i.e., $n \sim \mathcal{N}\left(0,\left(\sigma / P_{0}\right)^{2}\right)$.

\section{AN ACHIEVABLE RATE REGION OF THE CHANNEL IN}

In this section, we derive an achievable rate region for the channel in (5) using the ZF precoder. For the $2 \times 2 \mathrm{MU}-\mathrm{MISO}$ system discussed in section II, the ZF precoding matrix is uniquely given by $\boldsymbol{A}=P_{0} \boldsymbol{H}^{-1}$, i.e., $\boldsymbol{x}^{\prime}=x / P_{0}=\boldsymbol{A} u / P_{0}=$ $P_{0} \boldsymbol{H}^{-1} u / P_{0}=\boldsymbol{H}^{-1} \boldsymbol{u}$. Thus the received signal vector is given by

$$
\boldsymbol{y}=\boldsymbol{H} \boldsymbol{x}^{\prime}+\boldsymbol{n}=\boldsymbol{H} \boldsymbol{H}^{-1} \boldsymbol{u}+\boldsymbol{n}=\boldsymbol{u}+\boldsymbol{n} .
$$

i.e., there is no multi-user interference (MUI). Since

$$
\boldsymbol{u}=\boldsymbol{H} \boldsymbol{x}^{\prime}=\left[\begin{array}{ll}
\boldsymbol{h}_{1} & \boldsymbol{h}_{2}
\end{array}\right]\left[x_{1}^{\prime} x_{2}^{\prime}\right]^{T},
$$

\footnotetext{
${ }^{4}$ In subsequent discussions, by "received electrical signal", we refer to the "normalized received electrical signal".

${ }^{5}$ Note that $h_{k i}$ 's are non negative and model the overall gains of the line of sight (LOS) optical path between the $i^{\text {th }}$ LED and the $k^{\text {th }}$ user and also the responsivity of the PD of the $k^{t h}$ user [3].

${ }^{6}$ Note that the above noise impairments of the received signal are the main impairments that are commonly assumed in VLC systems [3].
} 
and $0 \leq x_{i}^{\prime} \leq 1, i=1,2$ (see (4)) it follows that, the information signal vector $\boldsymbol{u}$ must be limited to the region

$$
R_{/ /}(\boldsymbol{H}) \triangleq\left\{\boldsymbol{u} \mid \boldsymbol{u}=\boldsymbol{H} \boldsymbol{x}^{\prime}, \quad 0 \leq x_{1}^{\prime} \leq 1,0 \leq x_{2}^{\prime} \leq 1\right\} .
$$

The region $R_{/ /}(\boldsymbol{H})$ is a parallelogram with its two non parallel sides as $\boldsymbol{h}_{1}$ and $\boldsymbol{h}_{2}$ (see $R_{/ /}(\boldsymbol{H})$ in Fig. 2). In addition to this, the diagonal of the parallelogram $R_{/ /}(\boldsymbol{H})$ is the vector $\boldsymbol{h}_{1}+\boldsymbol{h}_{2}$ as shown in Fig. 2.

Let $E[\boldsymbol{u}] \triangleq\left[E\left[u_{1}\right] E\left[u_{2}\right]\right]^{T}$ be the mean information symbol vector. From (7) and (4), the mean information symbol vector is given by

$$
E[\boldsymbol{u}]=\boldsymbol{H} E\left[\boldsymbol{x}^{\prime}\right]=\left[\begin{array}{ll}
\boldsymbol{h}_{1} & \boldsymbol{h}_{2}
\end{array}\right] E\left[x_{1}^{\prime} x_{2}^{\prime}\right]^{T} \stackrel{(a)}{=} \xi\left(\boldsymbol{h}_{1}+\boldsymbol{h}_{2}\right),
$$

where step (a) follows from (5). Therefore, the mean information symbol pair $\left(E\left[u_{1}\right], E\left[u_{2}\right]\right)$ is a point corresponding to the tip of the vector $E[\boldsymbol{u}]=\xi\left(\boldsymbol{h}_{1}+\boldsymbol{h}_{2}\right)$. From (8) it is clear that the vector $\left(\boldsymbol{h}_{1}+\boldsymbol{h}_{2}\right)$ is a diagonal of $R_{/ /}(\boldsymbol{H})$ (see Fig. 2 ). For a given $0 \leq \xi \leq 1$, the tip of the mean information symbol vector $\xi\left(\boldsymbol{h}_{1}+\boldsymbol{h}_{2}\right)$ is therefore a fixed point on the diagonal $\left(\boldsymbol{h}_{1}+\boldsymbol{h}_{2}\right)$. We denote this point by

$$
\begin{aligned}
D(\boldsymbol{H}, \xi) & =\left(E\left[u_{1}\right], E\left[u_{2}\right]\right) \\
& =\left(\xi\left(h_{11}+h_{12}\right), \xi\left(h_{21}+h_{22}\right)\right) .
\end{aligned}
$$

With the ZF precoder, the broadcast channel in (5) is reduced to two parallel SISO (single-input single-output) optical channels between the transmitter and the two users (see (6)). Since $u_{1} \in \mathcal{U}_{1}$ and $u_{2} \in \mathcal{U}_{2}$ are independent and originate from different codebooks, it follows that $\left(u_{1}, u_{2}\right) \in \mathcal{U}_{1} \times \mathcal{U}_{2}$. From (8), we know that $\left(u_{1}, u_{2}\right)$ must belong to the parallelogram $R_{/ /}(\boldsymbol{H})$ and therefore

$$
\mathcal{U}_{1} \times \mathcal{U}_{2} \subset R_{/ /}(\boldsymbol{H}) .
$$

In general we choose $\mathcal{U}_{1}$ and $\mathcal{U}_{2}$ to be intervals of the type $[a, b]$ [9]. Let the length of the intervals $\mathcal{U}_{1}$ and $\mathcal{U}_{2}$ be $L_{1}$ and $L_{2}$ respectively, i.e. $\left|\mathcal{U}_{1}\right|=L_{1},\left|\mathcal{U}_{2}\right|=L_{2}$. With $\mathcal{U}_{1}$ and $\mathcal{U}_{2}$ as intervals, it is clear that $\mathcal{U}_{1} \times \mathcal{U}_{2}$ must be a rectangle whose length along the $u_{1}$ axis is $L_{1}$ and that along the $u_{2}$ axis is $L_{2}$. In this paper we assume $u_{1}$ and $u_{2}$, to be uniformly distributed in the interval $\mathcal{U}_{1}$ and $\mathcal{U}_{2}$ respectively. ${ }^{7}$ Therefore, for a given $\mathcal{U}_{1}$ and $\mathcal{U}_{2}$, the mean information symbol pair $\left(E\left[u_{1}\right], E\left[u_{2}\right]\right)$ will lie at the point of intersection of the two diagonal of the rectangle $\mathcal{U}_{1} \times \mathcal{U}_{2}$. We will subsequently call this point of intersection as the "midpoint" of the rectangle $\mathcal{U}_{1} \times \mathcal{U}_{2}$ and will denote it by $\mathscr{C}\left(\mathcal{U}_{1}, \mathcal{U}_{2}\right)$.

From (10), it follows that the mean information symbol pair must exactly coincide with $D(\boldsymbol{H}, \xi)$, i.e.

$$
\mathscr{C}\left(\mathcal{U}_{1}, \mathcal{U}_{2}\right)=D(\boldsymbol{H}, \xi)
$$

The ZF precoder transforms the broadcast channel into two parallel SISO channels $y_{i}=u_{i}+n_{i}, u_{i} \in \mathcal{U}_{i}, i=1,2$. Let $R_{1}$ and $R_{2}$ denote the information rates achieved on these SISO channels with $u_{i}$ distributed uniformly in $\mathcal{U}_{i}$. Any given $\mathcal{U}_{1}$ and $\mathcal{U}_{2}$ satisfying the conditions in (11) and (12) would satisfy the optical power constraints in (4) and would therefore

\footnotetext{
${ }^{7}$ At high $\operatorname{SNR}\left(P_{0} / \sigma>>1\right)$, uniformly distributed information symbol is near capacity achieving [10].
}

correspond to an achievable rate pair for the broadcast channel in (5). Since a rectangle in the $u_{1}-u_{2}$ plane corresponds to a unique $\mathcal{U}_{1}$ and $\mathcal{U}_{2}$ and vice versa, it follows that any rectangle lying inside the parallelogram $R_{/ /}(\boldsymbol{H})$ and having its midpoint at $D(\boldsymbol{H}, \xi)$ will correspond to an achievable rate pair. In this paper, for the broadcast channel in (5), we therefore propose an achievable rate region which consists of rate pairs corresponding to such rectangles (one such rectangle is shown in Fig. 2). We define our proposed rate region more precisely in the following. Towards this end, we first formally define the achievable rate of a SISO AWGN optical channel, where the transmitted information symbol is constrained to lie in an interval.

Result 1. [From [6], [10]] The achievable information rate of a SISO channel $y=u+n$ (where $u \sim U n i f[a, b]$ and $n \sim \mathcal{N}\left(0,\left(\sigma / P_{0}\right)^{2}\right)$ depends on the interval $[a, b]$ only through its length $L=|b-a|$, and is given by the function

$$
C\left(L=|b-a|, P_{0} / \sigma\right) \triangleq I(u ; y),
$$

here Unif $[a, b]$ denote the uniform distribution in the interval $[a, b]$ and $I(u ; y)$ is the mutual information between $u$ and $y$.

Result 2. [From [6], [10]] The function $C\left(L, P_{0} / \sigma\right)$ is continuous with respect to $L$ and increases monotonically with increasing $L$ for a fixed $P_{0} / \sigma$.

Let $\operatorname{Rect}\left(L_{1}, L_{2}, D(\boldsymbol{H}, \xi)\right)$ denote the unique rectangle having its midpoint as $D(\boldsymbol{H}, \xi)$ and whose length along the $u_{1}$ axis is $L_{1}$ and that along the $u_{2}$ axis is $L_{2}$ (see Fig. 2). Any such rectangle $\operatorname{Rect}\left(L_{1}, L_{2}, D(\boldsymbol{H}, \xi)\right) \subset R_{/ /}(\boldsymbol{H})$ will correspond to an achievable rate pair given by

$$
\left(R_{1}, R_{2}\right) \triangleq\left(C\left(L_{1}, P_{0} / \sigma\right), C\left(L_{2}, P_{0} / \sigma\right)\right)
$$

For a given $\left(\boldsymbol{H}, P_{0} / \sigma, \xi\right)$ the proposed achievable rate region for the $\mathrm{ZF}$ precoder is given by

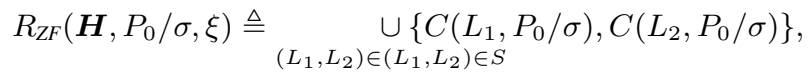

where $S \triangleq\left\{\left(L_{1} \geq 0, L_{2} \geq 0\right) \mid \exists \operatorname{Rect}\left(L_{1}, L_{2}, D(\boldsymbol{H}, \xi)\right)\right.$ $\left.\subset R_{/ /}(\boldsymbol{H})\right\}$.

\section{Characterizing the Boundary of the Rate REGION $R_{\mathrm{ZF}}\left(\boldsymbol{H}, P_{0} / \sigma, \xi\right)$}

In this section, we completely characterize the boundary of the rate region, $R_{Z F}\left(\boldsymbol{H}, P_{0} / \sigma, \xi\right)$, for a fixed $\left(\boldsymbol{H}, P_{0} / \sigma, \xi\right)$. Towards this end, for each information rate $R_{1}$ achievable by the first user, we find the corresponding maximum possible information rate $R_{2}$ achievable by the second user. Each pair of $R_{1}$ and its corresponding maximum possible $R_{2}$ is therefore a point on the boundary of the proposed rate region. By increasing $R_{1}$ from 0 to its maximum possible value, all such $\left(R_{1}, R_{2}\right)$ pairs characterize the boundary of the rate region.

From (15), we know that any achievable rate pair $\left(R_{1}, R_{2}\right)$ in the proposed rate region $R_{Z F}\left(\boldsymbol{H}, P_{0} / \sigma, \xi\right)$ corresponds to some rectangle $\operatorname{Rect}\left(L_{1}, L_{2}, D(\boldsymbol{H}, \xi)\right)$. The rate to the $i^{\text {th }}$ user, i.e. $R_{i}=C\left(L_{i}, P_{0} / \sigma\right), i=1,2$ depends only on the length of this rectangle along the $u_{i}$-axis. Since the 
$C\left(L, P_{0} / \sigma\right)$ function is monotonic and continuous in its first argument, each value of $R_{i}$ corresponds to a unique $L_{i}$ and vice versa. Therefore, towards characterizing the boundary of $R_{Z F}\left(\boldsymbol{H}, P_{0} / \sigma, \xi\right)$, we note that for a given $R_{1}$,i.e., for a given length $L_{1}$ along the $u_{1}$-axis, we would like to find the largest possible $R_{2}$,i.e., the largest possible $L_{2}$ such that the rectangle $\operatorname{Rect}\left(L_{1}, L_{2}, D(\boldsymbol{H}, \xi)\right)$ lies entirely inside $R_{/ /}(\boldsymbol{H})$. Hence, we can characterize the boundary of $R_{Z F}\left(\boldsymbol{H}, P_{0} / \sigma, \xi\right)$ simply by varying $L_{1}=x$ from 0 to its maximum possible value (denoted by $L_{1}^{\max }(\xi)$ ), and for each value of $L_{1}=x \in\left[0, L_{1}^{\max }(\xi)\right]$ we find the largest possible $L_{2}=L_{2}^{\xi}(x)$ which gives us a corresponding rate pair $\left(R_{1}, R_{2}\right)=\left(C\left(L_{1}=x, P_{0} / \sigma\right), C\left(L_{2}=L_{2}^{\xi}(x), P_{0} / \sigma\right)\right)$ on the boundary of the rate region $R_{Z F}\left(\boldsymbol{H}, P_{0} / \sigma, \xi\right)$.

For a given $\left(L_{1}=x, L_{2}=L_{2}^{\xi}(x)\right)$ the corresponding information rate pair lies on the boundary of the proposed rate region $R_{Z F}\left(\boldsymbol{H}, P_{0} / \sigma, \xi\right)$. We denote this information rate pair by $\left(R_{1}^{B d}\left(x, P_{0} / \sigma, \xi\right), R_{2}^{B d}\left(x, P_{0} / \sigma, \xi\right)\right)$. From (14), this information rate pair is given by

$$
\begin{gathered}
R_{1}^{B d}\left(x, P_{0} / \sigma, \xi\right) \triangleq C\left(L=x, P_{0} / \sigma\right) . \\
R_{2}^{B d}\left(x, P_{0} / \sigma, \xi\right) \triangleq C\left(L=L_{2}^{\xi}(x), P_{0} / \sigma\right) .
\end{gathered}
$$

This then completely characterizes the boundary of the rate region $R_{Z F}\left(\boldsymbol{H}, P_{0} / \sigma, \xi\right)$, which is given by ${ }^{8}$

$$
\begin{aligned}
R_{Z F}^{B d}\left(\boldsymbol{H}, P_{0} / \sigma, \xi\right) & \triangleq \cup_{0 \leq x \leq L_{1}^{\max }(\xi)}\left(R_{1}^{B d}\left(x, P_{0} / \sigma, \xi\right), R_{2}^{B d}\left(x, P_{0} / \sigma, \xi\right)\right) \\
& =\underset{0 \leq x \leq L_{1}^{\max }(\xi)}{\cup}\left(C\left(x, P_{0} / \sigma\right), C\left(L_{2}^{\xi}(x), P_{0} / \sigma\right)\right)
\end{aligned}
$$

It is noted that the analysis done in this paper is applicable to any placement of the users and the LEDs. Subsequently, we follow the following convention that, by LED 1 we shall refer to the LED whose channel vector has a higher inclination angle (from the $u_{1}$ axis) than the inclination angle of the channel vector of the other LED.

Let the inclination of the vector $\boldsymbol{h}_{1}$ and $\boldsymbol{h}_{2}$ from the $u_{1}$ axis be $\theta_{1}$ and $\theta_{2}$ respectively (see Fig. 2). From our definition of LED 1 and LED 2 (see the above paragraph), it follows that $\theta_{1}>\theta_{2}$. Therefore it follows that $\tan \theta_{1}>\tan \theta_{2}$. Since

$$
\tan \theta_{1}=h_{21} / h_{11}, \quad \tan \theta_{2}=h_{22} / h_{12} .
$$

Hence, $\tan \theta_{1}>\tan \theta_{2}$ implies that

$$
\begin{aligned}
h_{21} / h_{11}-h_{22} / h_{12} & >0, \\
h_{11} h_{22}-h_{12} h_{21} & <0, \quad \text { i.e. } \\
\operatorname{det}(\boldsymbol{H}) & <0
\end{aligned}
$$

In the following proposition, we first compute the maximum value of $L_{1}$ and subsequently we derive the maximum value of $L_{2}$ for each value of $L_{1}$.

Proposition 1. The largest possible value of $L_{1}$ (i.e., length of the interval $\mathcal{U}_{1}$ ) such that there exists a rectangle

\footnotetext{
${ }^{8}$ From (16) and (17) it is clear that the exact computation of $R_{1}^{B d}\left(x, P_{0} / \sigma, \xi\right)$ and $R_{2}^{B d}\left(x, P_{0} / \sigma, \xi\right)$ requires the computation of $L_{2}^{\xi}(x)$ for which we derive closed form expressions in the next section. Computation of $R_{1}^{B d}\left(x, P_{0} / \sigma, \xi\right)$ and $R_{2}^{B d}\left(x, P_{0} / \sigma, \xi\right)$ also requires us to compute the $C\left(L, P_{0} / \sigma\right)$ function which is done numerically.
}

$\operatorname{Rect}\left(L_{1}, L_{2}, D(\boldsymbol{H}, \xi)\right)\left(L_{2} \geq 0\right)$ which lies completely inside the parallelogram $R_{/ /}(\boldsymbol{H})$, is given by

$$
\begin{aligned}
L_{1}^{\max }(\xi) \triangleq & \max _{\substack{L_{1} \geq 0, L_{2} \geq 0 \\
\operatorname{Rect}\left(L_{1}, L_{2}, D(\boldsymbol{H}, \xi)\right) \subset R_{/ /}(\boldsymbol{H})}} L_{1} \\
& = \begin{cases}\frac{-2 \xi \operatorname{det}(\boldsymbol{H})}{\max \left(h_{21}, h_{22}\right)}, & 0 \leq \xi \leq 1 / 2 \\
\frac{-2(1-\xi) \operatorname{det}(\boldsymbol{H})}{\max \left(h_{21}, h_{22}\right)}, & 1 / 2 \leq \xi \leq 1 .\end{cases}
\end{aligned}
$$

Proof: See Appendix A.

It is clear from (21) in Proposition 1 that $L_{1}^{\max }(\xi)$ is a continuous function of $\xi$ and $L_{1}^{\max }(\xi)=L_{1}^{\max }(1-\xi)$.

Remark 1. The function $L_{1}^{\max }(\xi)$ is a continuous function of $\xi$ and is symmetric about $\xi=1 / 2$, i.e.

$$
L_{1}^{\max }(\xi)=L_{1}^{\max }(1-\xi), \quad 0 \leq \xi \leq 1
$$

From (21) it is clear that since $\operatorname{det}(\boldsymbol{H})<0$ (see (20)) $L_{1}^{\max }(x)$ is linearly increasing for $0 \leq \xi \leq 1 / 2$ and is linearly decreasing for $1 / 2 \leq \xi \leq 1$. Hence $L_{1}^{\max }(\xi)$ has a unique maximum at $\xi=1 / 2$.

Remark 2. The function $L_{1}^{\max }(\xi)$ has its unique maximum at $\xi=1 / 2$, i.e.

$$
\underset{0 \leq \xi \leq 1}{\arg \max } L_{1}^{\max }(\xi)=1 / 2
$$

Proposition 2. For a given $L_{1}=x \in\left[0, L_{1}^{\max }(\xi)\right]$, the largest possible $L_{2} \geq 0$ such that there exists a rectangle $\operatorname{Rect}\left(x, L_{2}, D(\boldsymbol{H}, \xi)\right) \subset R_{/ /}(\boldsymbol{H})$, is given by

$$
\begin{aligned}
L_{2}^{\xi}(x) & \triangleq \max _{\substack{L_{2} \geq 0 \\
\operatorname{Rect}\left(x, L_{2}, D(\boldsymbol{H}, \xi)\right) \subset \mathcal{R} / /(\boldsymbol{H})}} L_{2} \\
& =2 \min \left(L_{2}^{u p, \xi}(x), L_{2}^{\text {down }, \xi}(x)\right),
\end{aligned}
$$

where $L_{2}^{u p, \xi}(x)$ is given by

Case I: $0 \leq \xi \leq \frac{h_{11}}{h_{11}+h_{12}}$

$$
L_{2}^{u p, \xi}(x)=\left\{\frac{-\xi \operatorname{det}(\boldsymbol{H})-\frac{x}{2} h_{21}}{h_{11}}, \quad 0 \leq x \leq L_{1}^{\max }(\xi)\right.
$$

Case II: $\frac{h_{11}}{h_{11}+h_{12}} \leq \xi \leq 1$

$$
L_{2}^{u p, \xi}(x)= \begin{cases}\frac{-(1-\xi) \operatorname{det}(\boldsymbol{H})-\frac{x}{2} h_{22}}{h_{12}}, & 0 \leq x \leq \eta_{3}(\xi) \\ \frac{-\xi \operatorname{det}(\boldsymbol{H})-\frac{x}{2} h_{21}}{h_{11}}, & \eta_{3}(\xi) \leq x \leq L_{1}^{\max }(\xi)\end{cases}
$$

where $\eta_{3}(\xi) \triangleq 2 \xi h_{12}-2(1-\xi) h_{11}$.

$L_{2}^{\text {down }, \xi}(x)$ is given by

Case I: $0 \leq \xi \leq \frac{h_{12}}{h_{11}+h_{12}}$

$$
L_{2}^{\text {down }, \xi}(x)= \begin{cases}\frac{-\xi \operatorname{det}(\boldsymbol{H})-\frac{x}{2} h_{22}}{h_{12}}, & 0 \leq x \leq \eta_{4}(\xi) \\ \frac{-(1-\xi) \operatorname{det}(\boldsymbol{H})-\frac{x}{2} h_{21}}{h_{11}}, & \eta_{4}(\xi) \leq x \leq L_{1}^{\max }(\xi)\end{cases}
$$

where $\eta_{4}(\xi) \triangleq 2(1-\xi) h_{12}-2 \xi h_{11}$. 
Case II: $\frac{h_{12}}{h_{11}+h_{12}} \leq \xi \leq 1$

$$
L_{2}^{\text {down }, \xi}(x)=\left\{\frac{-(1-\xi) \operatorname{det}(\boldsymbol{H})-\frac{x}{2} h_{21}}{h_{11}}, 0 \leq x \leq L_{1}^{\max }(\xi)\right.
$$

Proof: See Appendix B.

Lemma 1. The function $L_{2}^{\xi}(x)\left(0 \leq x \leq L_{1}^{\max }(\xi)\right)$ is a monotonically decreasing and continuous function of $x$.

Proof: From Proposition 2 it is clear that for a given $\xi$ both $L_{2}^{u p, \xi}(x)$ and $L_{2}^{\text {down, } \xi}(x)$ are continuous and monotonically decreasing function of $x$. From this it follows that $L_{2}^{\xi}(x)=2 \min \left(L_{2}^{u p, \xi}(x), L_{2}^{\text {down }, \xi}(x)\right)$ is a continuous and decreases monotonically with increasing $x$.

Lemma 2. The proposed rate region boundary $R_{Z F}^{B d}\left(\boldsymbol{H}, P_{0} / \sigma, \xi\right)$ is Pareto-optimal. That is, for any two rate pairs $(a, b)$ and $\left(a^{\prime}, b^{\prime}\right)$ on the boundary $R_{Z F}^{B d}\left(\boldsymbol{H}, P_{0} / \sigma, \xi\right)$, if $a^{\prime} \geq a$ then it must be true that $b^{\prime} \leq b$ and if $b^{\prime} \leq b$ then it must be true that $a^{\prime} \geq a$.

Proof: Let $(a, b)$ and $\left(a^{\prime}, b^{\prime}\right)$ be any two rate pairs on the boundary $R_{Z F}^{B d}\left(\boldsymbol{H}, P_{0} / \sigma, \xi\right)$ such that $a^{\prime} \geq a$. Then from $((16)$ and (17)) it follows that there exists $0 \leq$ $x \leq L_{1}^{\max }(\xi)$ and $0 \leq x^{\prime} \leq L_{1}^{\max }(\xi)$ such that $a=$ $C\left(x, P_{0} / \sigma\right), b=C\left(L_{2}^{\xi}(x), P_{0} / \sigma\right)$ and $a^{\prime}=C\left(x^{\prime}, P_{0} / \sigma\right)$, $b^{\prime}=C\left(L_{2}^{\xi}\left(x^{\prime}\right), P_{0} / \sigma\right)$, where the functions $C\left(x, P_{0} / \sigma\right)$ is defined in (13). From Result (2), we know that for a given $P_{0} / \sigma, C\left(x, P_{0} / \sigma\right)$ is a continuous and monotonically increasing function of its first argument. Since $C\left(x^{\prime}, P_{0} / \sigma\right)=$ $a^{\prime} \geq a=C\left(x, P_{0} / \sigma\right)$, it follows that $x^{\prime} \geq x$. From Lemma 1 , we know that $L_{2}^{\xi}(x)$ is a monotonically decreasing function of $x$, and therefore $L_{2}^{\xi}\left(x^{\prime}\right) \leq L_{2}^{\xi}(x)$, and hence $b^{\prime}=C\left(L_{2}^{\xi}\left(x^{\prime}\right), P_{0} / \sigma\right) \leq C\left(L_{2}^{\xi}(x), P_{0} / \sigma\right)=b$. Similarly, it can also be shown that, if $b^{\prime} \leq b$ then it must be true that $a^{\prime} \geq a$. This completes the proof.

Lemma 3. For a given $0 \leq \xi \leq 1$ and $x \in\left[0, L_{1}^{\max }(\xi)\right]$, the function $L_{2}^{\xi}(x)$ is symmetric about $\xi=1 / 2$, i.e.

$$
L_{2}^{\xi}(x)=L_{2}^{1-\xi}(x), \quad 0 \leq \xi \leq 1, x \in\left[0, L_{1}^{\max }(\xi)\right] .
$$

Proof: See Appendix C.

Using Lemma 3 along with the definition of the rate region boundary in (18) we get the following result.

Result 3. The proposed rate region boundary $R_{Z F}^{B d}\left(\boldsymbol{H}, P_{0} / \sigma, \xi\right)$ is symmetric about $\xi=1 / 2$, i.e.

$$
R_{Z F}^{B d}\left(\boldsymbol{H}, P_{0} / \sigma, \xi\right)=R_{Z F}^{B d}\left(\boldsymbol{H}, P_{0} / \sigma,(1-\xi)\right), \forall \xi \in[0,1] .
$$

The following theorem shows that for $0 \leq \xi \leq 1$, the largest rate region is achieved when $\xi=1 / 2$.

Theorem 1. For a fixed $\xi \in[0,1]$,

$$
R_{Z F}\left(\boldsymbol{H}, P_{0} / \sigma, \xi\right) \subseteq R_{Z F}\left(\boldsymbol{H}, P_{0} / \sigma, 1 / 2\right) .
$$

Proof: See Appendix D.

The proposed rate region boundary $R_{Z F}^{B d}\left(\boldsymbol{H}, P_{0} / \sigma, \xi\right)$ can be used to compute many practical operating points. Consider a case where we are interested in finding the largest achievable rate pair $\left(R_{1}, R_{2}\right)$ such that $R_{2}=\alpha R_{1}$. This operating point could make sense, if for example the average data throughput requested by user 2 is $\alpha$ times that of the throughput requested by user 1.

Moreover, for a given $\alpha>0$ and $P_{0} / \sigma$, the maximum achievable rate pair of the form $(r, \alpha r)$ is given by $\left(R_{\max }^{\alpha}(\xi), \alpha R_{\max }^{\alpha}(\xi)\right)$ where $R_{\max }^{\alpha}(\xi)$ is defined as

$$
R_{\max }^{\alpha}(\xi) \triangleq \max _{r \mid(r, \alpha r) \in R_{Z F}\left(\boldsymbol{H}, P_{0} / \sigma, \xi\right)} r .
$$

Theorem 2. $R_{\max }^{\alpha}(\xi)$ is unique and $\left(R_{\max }^{\alpha}(\xi), \alpha R_{\max }^{\alpha}(\xi)\right)$ lies on the boundary $R_{Z F}^{B d}\left(\boldsymbol{H}, P_{0} / \sigma, \xi\right)$.

Proof: See Appendix E.

Remark 3. From the proof in Appendix $E$ it is clear that Theorem 2 is non-trivial as it depends on the monotonicity and continuity of $L_{2}^{\xi}(x)$, which is shown in Lemma 1. If Lemma 1 were not true, Theorem 2 would not hold.

Result 4. Using Theorem 2 and (30) of Result 3 it follows that for a given $\alpha>0, R_{\max }^{\alpha}(\xi)$ is symmetric about $\xi=1 / 2$, i.e.

$$
R_{\max }^{\alpha}(\xi)=R_{\max }^{\alpha}(1-\xi), \quad \forall \alpha>0, \xi \in[0,1] .
$$

Corollary 2.1. From the geometrical interpretation of Theorem 2 it follows that $\left(R_{\max }^{\alpha}(\xi), \alpha R_{\max }^{\alpha}(\xi)\right)$ lies on the intersection of the straight line $R_{2}=\alpha R_{1}$ and the rate region boundary $R_{Z F}^{B d}\left(\boldsymbol{H}, P_{0} / \sigma, \xi\right)$. Further, from the Paretooptimality of the proposed rate region boundary, it follows that there is only a unique point of intersection between the line $R_{2}=\alpha R_{1}$ and $R_{Z F}^{B d}\left(\boldsymbol{H}, P_{0} / \sigma, \xi\right)$.

\section{A. Maximum symmetric rate $R^{\text {sym }}(\xi)$}

Note that for the special case of $\alpha=1, R_{\max }^{\alpha}(\xi)$ is nothing but the maximum achievable symmetric rate which we shall denote by

$$
R^{s y m}(\xi) \triangleq R_{\max }^{\alpha=1}(\xi) .
$$

From Theorem 2 it is clear that the maximum symmetric rate is nothing but the largest rate $R$ such that the rate pair $(R, R)$ lies on the boundary $R_{Z F}^{B d}\left(\boldsymbol{H}, P_{0} / \sigma, \xi\right)$. From the characterization of the boundary points in (18), it follows that there must exist $\left(x, L_{2}^{\xi}(x)\right)$ for some $0 \leq x \leq L_{1}^{\max }(\xi)$ such that

$$
R=C\left(x, P_{0} / \sigma\right), \text { and } R=C\left(L_{2}^{\xi}(x), P_{0} / \sigma\right)
$$

and therefore

$$
x=L_{2}^{\xi}(x)
$$

since from Result 2 we know that $C\left(L, P_{0} / \sigma\right)$ is a continuous and monotonic function. From 14 it follows that there exists a rectangle $\operatorname{Rect}\left(x, L_{2}^{\xi}(x), D(\boldsymbol{H}, \xi)\right) \subset R_{/ /}(\boldsymbol{H})$ corresponding to the rate pair $(R, R)$ where $x$ satisfies 36 .

Since $x=L_{2}^{\xi}(x)$ it follows that this rectangle is infact a square. Further, from the definition of $L_{2}^{\xi}(x)$ in (24) it follows that this is the largest sized square whose midpoint is at $D(\boldsymbol{H}, \xi)$ and has side length $x$. 


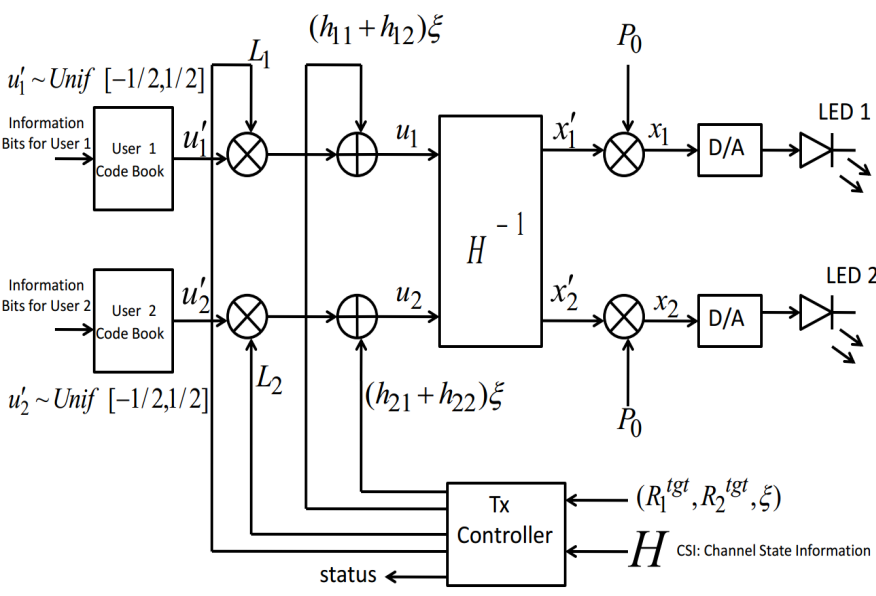

(a) Transmitter (Tx) block diagram.

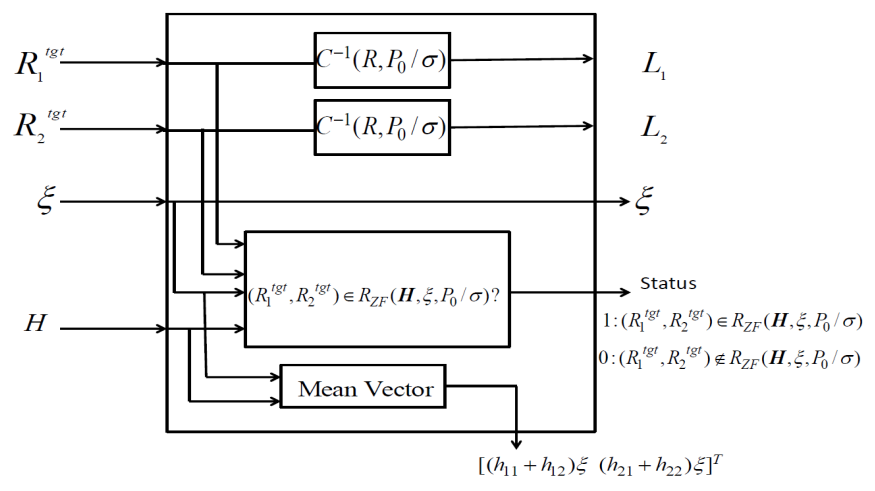

(b) Tx Controller block diagram.

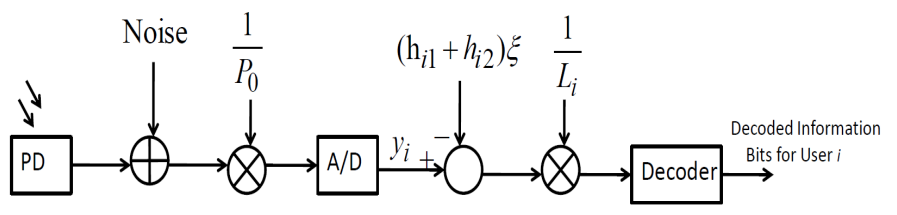

(c) Receiver block diagram for $i^{t h}$ user, $i=1,2$.

Fig. 3: A novel transceiver architecture for the proposed $2 \times 2$ MU-MISO VLC system with dimming Target of $\xi$ and target rate pair $\left(R_{1}^{t g t}, R_{2}^{t g t}\right)$.

Hence, the maximum achievable symmetric rate corresponds to the largest sized square which is completely inside $R_{/ /}(\boldsymbol{H})$ and has its midpoint at $D(\boldsymbol{H}, \xi)$.

\section{A Novel Transceiver Architecture}

In this section we propose a novel transceiver architecture for the practical implementation of the proposed $2 \times 2 \mathrm{MU}$ MISO VLC system to achieve any rate pair $\left(R_{1}, R_{2}\right) \in$ $R_{Z F}\left(\boldsymbol{H}, P_{0} / \sigma, \xi\right)$ (see Section IV), under a per-LED peak power constraint of $P_{0}$ and a controllable dimming target.

In Fig. 3, we have shown the block diagram of both the transmitter and the receiver. The block diagram in Fig. 3(a) depicts the transmitter, the block diagram in Fig. 3(b) depicts the controller for the transmitter which we call as Tx controller and the block diagram in Fig. 3(c) depicts the receiver. The working of this transceiver is as follows.

Consider a scenario where the rate requested by User 1 and User 2 are $R_{1}^{t g t}$ bpcu and $R_{2}^{t g t}$ bpcu respectively and to satisfy the lighting requirement inside the room the required dimming target is $\xi$. We call this rate pair $\left(R_{1}^{t g t}, R_{2}^{t g t}\right)$, as the target rate pair of the system. The Tx controller first checks if this target rate pair lies in the proposed achievable rate region $R_{Z F}\left(\boldsymbol{H}, P_{0} / \sigma, \xi\right)$ (see Section IV). If the target rate pair lies inside the proposed achievable rate region, (i.e., $\left.\left(R_{1}^{t g t}, R_{2}^{t g t}\right) \in R_{Z F}\left(\boldsymbol{H}, P_{0} / \sigma, \xi\right)\right)$ then the Tx controller flags 1 , otherwise it flags 0 (see status output of the Tx controller in Fig. 3(b)). If this flag is 1, then the Tx controller provides $L_{1}$ and $L_{2}$, the lengths of the intervals $\mathcal{U}_{1}$ and $\mathcal{U}_{2}$. From (15) we know that since $\left(R_{1}^{t g t}, R_{2}^{t g t}\right) \in R_{Z F}\left(\boldsymbol{H}, P_{0} / \sigma, \xi\right)$, there must exist some $\left(L_{1}, L_{2}\right)$ such that $R_{1}^{t g t}=C\left(L_{1}, P_{0} / \sigma\right)$ and $R_{2}^{\text {tgt }}=C\left(L_{2}, P_{0} / \sigma\right)$. From Result 2 we also know that for a given $P_{0} / \sigma, C\left(x, P_{0} / \sigma\right)$ is a monotonic function of $x$, and therefore there exists a corresponding inverse function $C^{-1}\left(R, P_{0} / \sigma\right)$ such that $C^{-1}\left(C\left(L, P_{0} / \sigma\right), P_{0} / \sigma\right)=L$ and $C\left(C^{-1}\left(R, P_{0} / \sigma\right), P_{0} / \sigma\right)=R$. It then follows $L_{i}=$ $C^{-1}\left(R_{i}^{t g t}, P_{0} / \sigma\right), i=1,2$ see Fig. 3(c). In the Tx controller we also have a block which outputs the mean information symbol vector $\xi\left(\boldsymbol{h}_{1}+\boldsymbol{h}_{2}\right)=\left[\begin{array}{lll}\xi\left(h_{11}+h_{12}\right) & \xi\left(h_{21}+h_{22}\right)\end{array}\right]^{T}$ (defined in (9)).

Further, in Fig. 3(a) the information bits for user 1 and user 2 are coded separately using independent codebooks each having i.i.d. codeword symbols which are unifromly distributed in $[-1 / 2,1 / 2]$. The codeword symbols for user 1 and user 2 are denoted by $u_{1}^{\prime}$ and $u_{2}^{\prime}$ respectively (note that $u_{1}$ and $u_{2}$ are the information symbols for User 1 and User 2 respectively). From Section III, we know that the information symbols for the $i^{t h}$ user must be uniformly distributed in the interval $\mathcal{U}_{i}$ i.e., $u_{i} \in \mathcal{U}_{i}=\left[\xi\left(h_{i 1}+h_{i 2}\right)-L_{i} / 2, \xi\left(h_{i 1}+h_{i 2}\right)+L_{i} / 2\right]$ (since the horizontal length of the rectangle corresponding to the rate pair $\left(R_{1}^{t g t}, R_{2}^{t g t}\right)$ is $L_{1}$, the vertical length of this rectangle is $L_{2}$ and its midpoint is $D(\boldsymbol{H}, \xi)$ ). Therefore, starting with the codeword symbol $u_{i}^{\prime}$ we can get the information symbol $u_{i}$ by

$$
u_{i}=L_{i} u_{i}^{\prime}+\xi\left(h_{i 1}+h_{i 2}\right), i=1,2 .
$$

This is also shown in Fig. 3. The information vector $\left[\begin{array}{ll}u_{1} & u_{2}\end{array}\right]^{T}$ is then precoded with $H^{-1}$ and scaled by $P_{0}$ to give the transmit signal vector $\left[x_{1} x_{2}\right]^{T}$. It is noted that the proposed transmitter architecture in Fig. 3(a) allows us to use the same channel encoder/codebook irrespective of the dimming target $\xi$. This is because the effect of the dimming control is only in shifting the mean of the information symbols $\left(u_{1}, u_{2}\right)$ (see the adders in Fig 3(a)). ${ }^{9}$

At the receiver after performing the operations shown in Fig. 3(c), we obtain the received vector as given by (5).

\section{NumericAl Results AND Discussions}

In this section, we present numerical results in support of the results reported in previous sections. For all numerical results we consider an indoor office room environment where

\footnotetext{
${ }^{9}$ Note that different target rates can be achieved by the same codebook through puncturing of the codewords.
} 
TABLE I: System Parameters used for Simulation

\begin{tabular}{|c|c|}
\hline PD area & $1 \mathrm{~cm}^{2}$ \\
\hline Receiver Field of Veiw (FOV) & $60[\mathrm{deg}]$. \\
\hline Refractive index of a lens at the PD & 1.5 \\
\hline Semi-angle at half power & 70 [deg.] \\
\hline
\end{tabular}

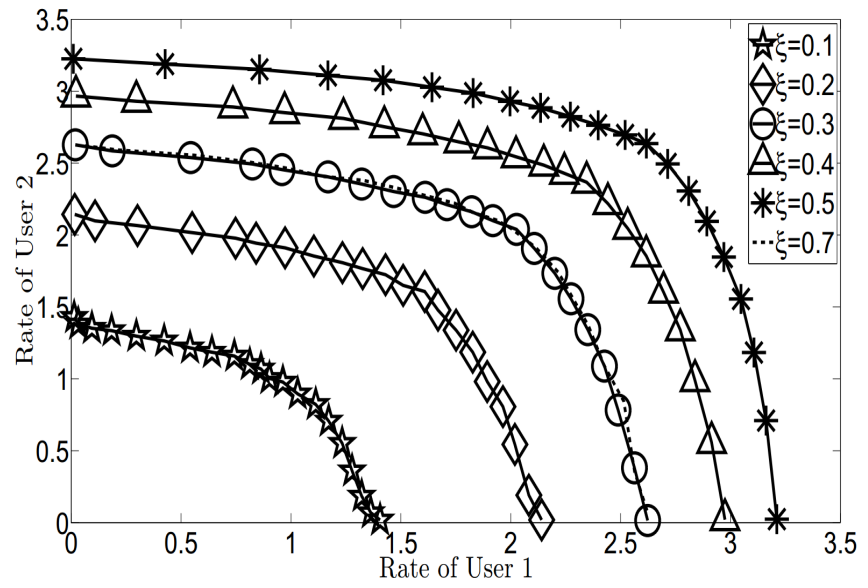

Fig. 4: Rate region boundary, $R_{Z F}^{B d}\left(\boldsymbol{H}, P_{0} / \sigma, \xi\right)$ for different values of dimming target, $\xi$.

the room is $5 \mathrm{~m} \times 5 \mathrm{~m}$ and its height is $3 \mathrm{~m}$. The two LEDs are attached to the ceiling and the two PDs (users) are placed at a height of $50 \mathrm{~cm}$ from the floor of the room. The two LEDs and the PDs lie in a plane perpendicular to the floor of the plane. The LEDs are placed $60 \mathrm{~cm}$ apart and the ratio $\frac{P_{0}}{\sigma}$ is fixed to $70 \mathrm{~dB}$. The channel gains are modeled for an indoor line of sight (LOS) channel. The other parameters used for simulation are given in Table I. All these parameters and the channel model are taken from prior work [3], [14], [17], [18].

In Fig. 4, for a LED separation of $0.6 \mathrm{~m}$ and PD (user) separation of $4 \mathrm{~m}$ such that the placement of both the LEDs and the PDs is symmetric ${ }^{10}$, we plot the proposed rate region boundary $R_{Z F}^{B D}\left(\boldsymbol{H}, P_{0} / \sigma, \xi\right)$ (see 18 ), for $\xi=0.1,0.2,0.3,0.4,0.5,0.7$.

For a given $\xi$, it is observed that the boundary is indeed Pareto-optimal as is stated in Lemma 2. We also observe that as $\xi$ increases from $\xi=0.1$ to $\xi=0.5$, the rate region expands and then it shrinks with further increase in $\xi$ from $\xi=0.5$ onwards to $\xi=1$. We have also observed that rate region boundary is same for both $\xi=0.3$ and $\xi=1-0.3=0.7$ as is stated in Result 3 (see the dotted line and the solid line marked with circle in Fig. 4). It is also observed that $\xi=1 / 2$ gives us the largest rate region as is stated in Theorem 1. The expansion/shrinking of the rate region with changing $\xi$ is explained in the following.

For a given $\xi$, the points on the rate region boundary correspond to rectangles in the $u_{1}-u_{2}$ plane having their midpoints at $D(\boldsymbol{H}, \xi)$, i.e., on the diagonal $\left(\boldsymbol{h}_{1}+\boldsymbol{h}_{2}\right)$ and at a distance of $\xi\left\|\boldsymbol{h}_{1}+\boldsymbol{h}_{2}\right\|$ from the origin. As $\xi$ increases, the midpoint of the rectangles move away from the origin

\footnotetext{
${ }^{10}$ Both the line segment joining the two users and the line segment joining the two LEDs have the same perpendicular bisector.
}

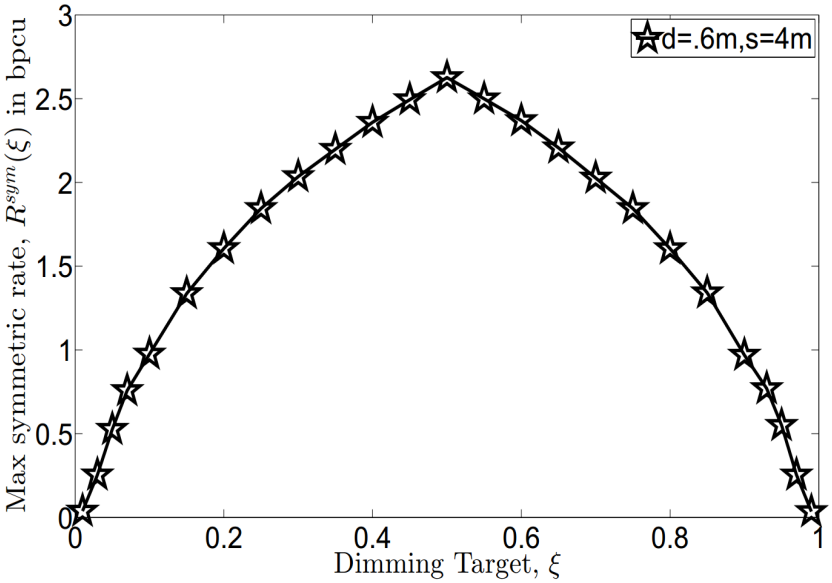

Fig. 5: Plot between Maximum Symmetric Rate and dimming Target, $\xi$.

and towards the interior of the parallelogram $R_{/ /}(\boldsymbol{H})$. This allows us to fit bigger rectangles and hence the rate region expands. As $\xi$ is increased beyond $\xi=0.5$ the midpoint of the rectangles moves towards the other end of the diagonal $\left(\boldsymbol{h}_{1}+\boldsymbol{h}_{2}\right)$ and hence the size of the rectangles reduces thereby shrinking the rate region.

In Fig. 5, for a fixed user separation of $s=4 \mathrm{~m}$, an LED separation of $d=60 \mathrm{~cm}$ and symmetric placement of LEDs and PDs, we plot the maximum achievable symmetric rate $R^{s y m}(\xi) \triangleq R_{\max }^{\alpha=1}(\xi)$ as a function of varying $\xi \in[0,1]$. We numerically find this operating point by considering all possible points in the $R_{1}-R_{2}$ plane which lie in the achievable rate region and also lie on the line $R_{2}=R_{1}$. Then among all these possible points we choose the one which has the largest component along the $R_{1}$ axis. From the figure it is observed that the variation in the maximum symmetric rate with change in the dimming target $\xi$ is small when $\xi$ is around $1 / 2$, as compared to when $\min (\xi, 1-\xi)$ is small. For example, when $\xi$ is reduced from $\xi=1 / 2$ to $\xi=0.4$ (i.e., $20 \%$ reduction), the corresponding maximum symmetric rate drops only by $11 \%$. However when $\xi$ is reduced by $20 \%$ from $\xi=0.07$ to $\xi=0.056$, the maximum symmetric rate decreases by approximately $25 \%$. From this it appears that the maximum symmetric rate is lesser sensitive to variations in the dimming target around $\xi=1 / 2$ as compared to variations around smaller values of $\xi$. It is also observed that symmetric rate $R^{\text {sym }}(\xi)$ is symmetric about $\xi=1 / 2$ as is stated in Result 4 (symmetric rate is nothing but $R_{\max }^{\alpha}(\xi)$ for $\alpha=1$ ).

We next study the variation in the maximum symmetric rate when the two users (PDs) are moved along a line parallel to the ceiling (at a height of $50 \mathrm{~cm}$ above the floor) while the two LEDs are stationary and fixed to the ceiling with a fixed separation of $60 \mathrm{~cm}$ between them and the dimming target is also fixed to $\xi=0.1$. Further, the two LEDs and the two PDs are co-planar. In Fig. 6, we plot the symmetric rate on the vertical axis as a function of the displacement ${ }^{11}$ of the two users from the origin (origin is the point of intersection of the

\footnotetext{
${ }^{11}$ Displacement is nothing but the distance of the user from the origin (see Fig. 1.
} 
Symmetric rate versus user displacement from the origin

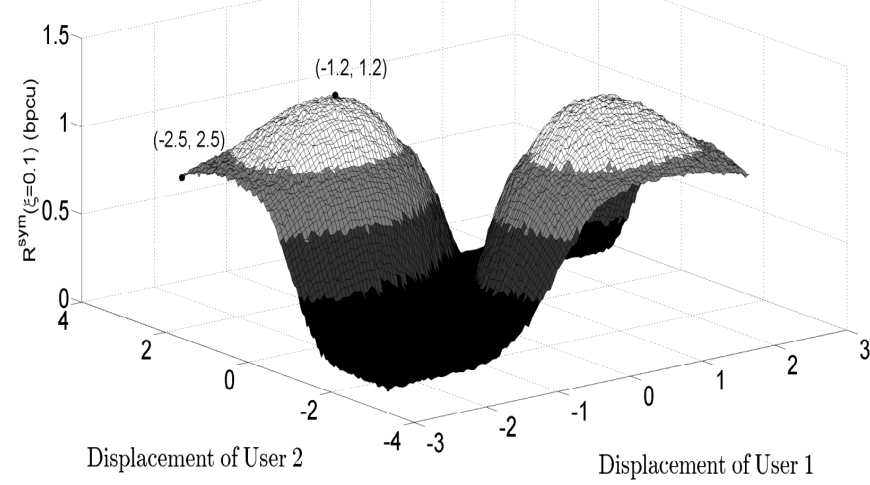

Fig. 6: Maximum symmetric rate vs displacement of the two users from the origin.

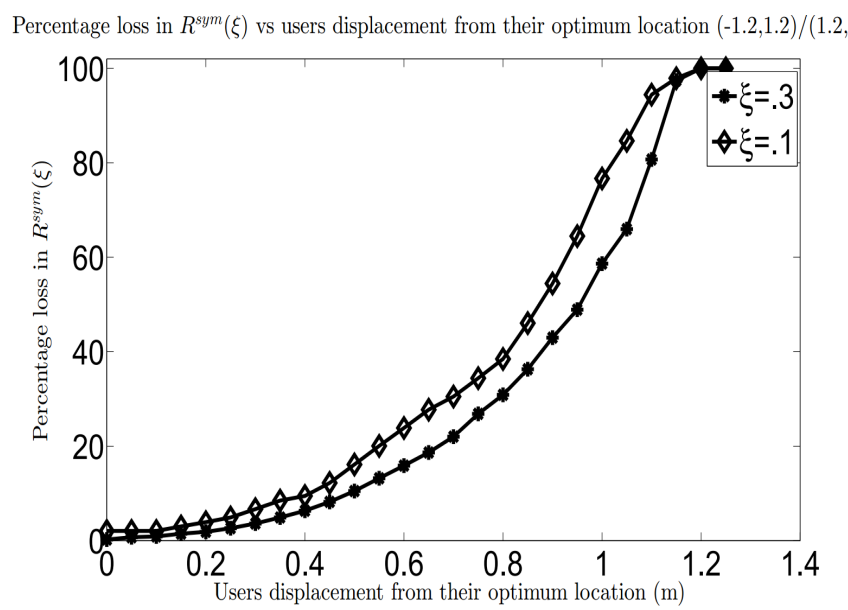

Fig. 7: Plot between percentage loss in $R^{\text {sym }}(\xi)$ and users displacement from their optimum location.

perpendicular bisector of the line joining the LEDs with the line joining the two users, see Fig . 1). In Fig. 6 a positive displacement implies that the user PD is located on the right side of the origin and vice versa (see Fig. 1).

It is observed that the maximum symmetric rate is almost zero if the displacement of both the users is same, i.e., the two users are almost co-located. In the figure this is represented by the dark black region. This is expected since in that case the channels to the users is also the same and hence the performance of the ZF precoder degrades. From the figure we observe that starting with both the users at the origin, as user 2 moves towards the right and User 1 moves towards left the maximum symmetric rate increases sharply (in the figure the colour changes from dark black to light black to gray to white as the displacement vector moves from $(0,0)$ to $(-1.2,1.2)$ ). This happens because as the users move away from each other, their channels become distinct i.e., the angles between the vectors $\boldsymbol{h}_{1}$ and $\boldsymbol{h}_{2}$ increases and hence the area of $R_{/ /}(\boldsymbol{H})$ increases. This results in an increase in the largest sized square that can be fit into $R_{/ /}(\boldsymbol{H})$ with center at $D(\boldsymbol{H}, \xi)$. This then implies that the maximum symmetric rate would also increase (see the discussion in Section IV-A for the correspondence between the largest square and the maximum symmetric rate). With further increase in the separation between the two users the angular separation between the channel vectors does not increase as sharply as before. At the same time, due to increased path loss from the LEDs to the users, the area of $R_{/ /}(\boldsymbol{H})$ starts decreasing which results in the decrease in the maximum symmetric rate. This can be seen in the figure, as the colour changes back from white to gray, as we move from the displacement vector $(-1.2,1.2)$ to $(-2.5,2.5)$. This shows that the maximum symmetric rate is dependent on the location of the users and therefore there is an optimal location ${ }^{12}$ for both the users which results in the highest symmetric rate. In Fig. 6 the optimum location is $(-1.2,1.2)$, or $(1.2,-1.2)$.

Next in Fig. 7, for a fixed LED separation of $60 \mathrm{~cm}$ we plot the percentage loss in the maximum symmetric rate $R^{\text {sym }}(\xi)$ (w.r.t. the symmetric rate at the optimum location) with the users' displacement from their optimum location for two different values of $\xi=0.1,0.3$.

It is observed that the percentage loss increases with increasing displacement of the PDs from their optimal location. Further, the increase in the percentage loss is small when the displacement is small as compared to when the displacement is large. For example, with $\xi=0.3$, the percentage loss increases only by $6 \%$ as the displacement increases from $0 \mathrm{~cm}$ to 40 $\mathrm{cm}$. However with a further increase in displacement from 40 $\mathrm{cm}$ to $80 \mathrm{~cm}$, the percentage loss increases sharply from $6 \%$ to $30 \%$. A similar behavior is also observed with $\xi=0.1$, though for a given displacement the loss is greater when $\xi=0.1$ as compared to when $\xi=0.3$. A practical application of this study could be in defining coverage zones for the PDs, i.e., the maximum allowable displacement for a fixed desired upper limit on the percentage loss. For example, in the current setup with $\xi=0.3$, for a $20 \%$ upper limit on the percentage loss, the maximum allowable displacement is roughly $70 \mathrm{~cm}$. It therefore appears that indoor VLC systems allow for a lot of flexibility in the movement of the user terminals without significant loss in the information rate.

\section{CONCLUSION}

We have proposed an achievable rate region for the $2 \times 2$ MU-MISO broadcast VLC channel under per-LED peak power constraint and dimming control. The boundary of the proposed rate region has been analytically characterized. We propose a novel transceiver architecture to implement such systems. Interestingly, the design of encoder/codebook is independent of the dimming target, which reduces the complexity of the transceiver. Work done in this paper reveals that, in an indoor setting, the two users have enough mobility around their optimal placement without sacrificing their information rates. Our work can also be applied to a 2-D setting, where the users are allowed to move in a plane rather than being restricted to a line.

\footnotetext{
${ }^{12} \mathrm{By}$ the optimum user location we mean the displacement vector of the users at which we get maximum $R^{\text {sym }}(\xi)$.
} 


\section{APPENDIX A}

\section{ProOF OF PROPOSITION 1}

Proof: Under the condition in (20), to find $L_{1}^{\max }(\xi)$ we need to consider three scenarios that cover all geometrically possible parallelograms $R_{/ /}(\boldsymbol{H})$ : (a) $\left(h_{11}<h_{12}\right.$ and $h_{21}>$ $\left.h_{22}\right)$; (b) $\left(h_{21} \leq h_{22}\right)$; and (c) $\left(h_{12} \leq h_{11}\right.$ and $\left.h_{21}>h_{22}\right)$.

For a given dimming target, $\xi$, let $L_{3}$ denote the length of the longest line segment parallel to the $u_{1}$-axis lying completely inside $R_{/ /}(\boldsymbol{H})$ and whose midpoint coincides with the point $D(\boldsymbol{H}, \xi)(D(\boldsymbol{H}, \xi)$ is defined in (10)). For any rectangle $\operatorname{Rect}\left(L_{1}, L_{2}, D(\boldsymbol{H}, \xi)\right) \subset R_{/ /}(\boldsymbol{H})$, its side along the $u_{1}$ axis is a line segment inside $R_{/ /}(\boldsymbol{H})$. From the definition of $L_{3}$, it follows that $L_{1} \leq L_{3}$ for any rectangle $\operatorname{Rect}\left(L_{1}, L_{2}, D(\boldsymbol{H}, \xi)\right) \subset R_{/ /}(\boldsymbol{H})$. Additionally, the longest line segment of length $L_{3}$ corresponds to a rectangle $\operatorname{Rect}\left(L_{3}, L_{2}=0, D(\boldsymbol{H}, \xi)\right) \subset R_{/ /}(\boldsymbol{H})$. Hence, it is clear that $L_{1}^{\max }(\xi)=L_{3}$, i.e.

$$
\begin{aligned}
L_{1}^{\max }(\xi) & \triangleq \max _{\substack{L_{1} \geq 0, L_{2} \geq 0 \\
\operatorname{Rect}\left(L_{1}, L_{2}, D(\boldsymbol{H}, \xi)\right) \subset R_{/ /}(\boldsymbol{H})}} L_{1} \\
& =\max _{\left\{L_{1}>0 \mid \operatorname{Rect}\left(L_{1}, L_{2}=0, D(\boldsymbol{H}, \xi)\right) \subset R_{/ /}(\boldsymbol{H})\right\}} L_{1}
\end{aligned}
$$

In the following, we firstly evaluate the expression for $L_{1}^{\max }(\xi)$ for scenario (a), i.e., when the channel gains satisfy $\left(h_{11}<h_{12}\right.$ and $\left.h_{21}>h_{22}\right)$. Towards this end, we partition $R_{/ /}(\boldsymbol{H})$ into three regions, Region $i, i=1,2,3$, as is shown in Fig. 8. We now derive an expression for $L_{1}^{\max }(\xi)$ depending upon the region where $D(\boldsymbol{H}, \xi)$ lies. In Fig. 8 we denote $D(\boldsymbol{H}, \xi)$ by the point $P$ if $D(\boldsymbol{H}, \xi)$ lies in Region 1, by the point $Q$ if $D(\boldsymbol{H}, \xi)$ lies in Region 2 and by the point $S$ if $D(\boldsymbol{H}, \xi)$ lies in Region 3. Next, we compute $L_{1}^{\max }(\xi)$ when $D(\boldsymbol{H}, \xi)$ lies in Region 1.

Computation of $L_{1}^{\max }(\xi)$ when $P=D(\boldsymbol{H}, \xi) \in$ Region 1 : The point $D(\boldsymbol{H}, \xi)$ belongs to Region 1 if and only if

$$
0 \leq O P \leq O T,
$$

where the point $T$ denote point of intersection of the diagonal $O B$ and $C C^{\prime}$. Further, the line $C C^{\prime}$ is the line parallel to the $u_{1}$-axis. Next, by looking at the right angle triangle $O T_{1} T$ in Fig. 8, it follows that $O T=T T_{1} / \sin \gamma$, where $\gamma$ denotes inclination of the diagonal, $O B$, of the parallelogram $R_{/ /}(\boldsymbol{H})$ from the $u_{1}$-axis. Since, from Fig. 8, $T T_{1}=h_{22}$, and $\sin \gamma=$ $\left(h_{21}+h_{22}\right) / O B$, it follows that

$$
O T=\frac{h_{22} O B}{h_{21}+h_{22}}
$$

Since the point $P$ is nothing but the point $D(\boldsymbol{H}, \xi)$, from (10), it follows that $O P=\xi O B$. Using $O P=\xi O B$ and (40) in (39) we have that $D(\boldsymbol{H}, \xi) \in$ Region 1 if and only if

$$
0 \leq \xi \leq \frac{h_{22}}{h_{21}+h_{22}},
$$

For all such values of the dimming target, $\xi$, satisfying (41), it follows that $D(\boldsymbol{H}, \xi) \in$ Region 1 . Next, we evaluate $L_{1}^{\max }(\xi)$ when $0 \leq \xi \leq h_{22} /\left(h_{21}+h_{22}\right)$.

Since $L_{1}^{\max }(\xi)$ is the length of the line segment parallel to the $u_{1}$-axis having its midpoint at point $P$ and lying

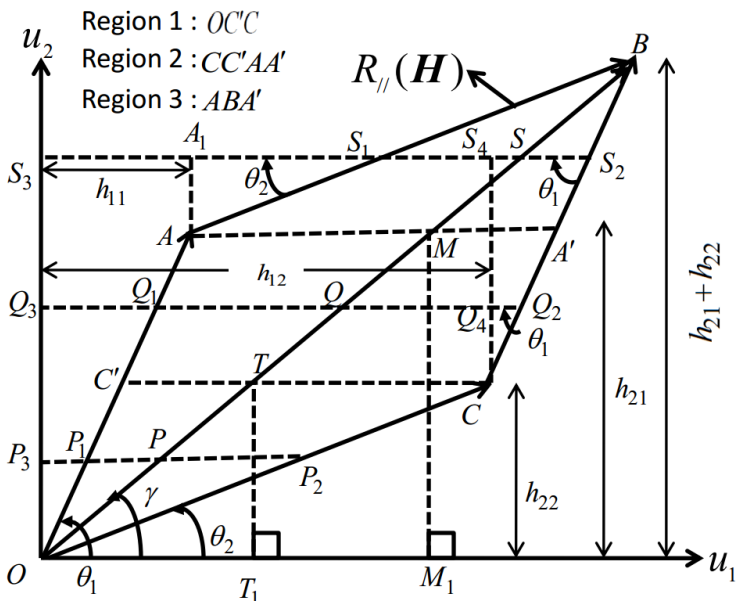

Fig. 8: Partition of the parallelogram $O A B C \triangleq R_{/ /}(\boldsymbol{H})$ into three different regions for the scenario $\left(h_{11}<h_{12}\right.$ and $h_{21}>$ $\left.h_{22}\right)$. Note that $A A^{\prime}$ and $C C^{\prime}$ are both parallel to the $u_{1}$ axis.

completely inside $R_{/ /}(\boldsymbol{H})$. it follows that

$$
L_{1}^{\max }(\xi)=2 \min \left(P P_{1}, P P_{2}\right),
$$

where both the line segments $P P_{1}$ and $P P_{2}$ are parallel to the $u_{1}$-axis. Further, $P_{1}$ lies on the line $O A$ whereas $P_{2}$ lies on the line $O C$ as shown in Fig. 8. Next, we evaluate $P P_{1}$ and $\mathrm{PP}_{2}$. To this end, from Fig. 8 , we compute the length of the line segment $P P_{1}$ as follows

$$
\begin{aligned}
P P_{1} & =P P_{3}-P_{1} P_{3} \\
& \stackrel{(a)}{=} \xi\left(h_{11}+h_{12}\right)-O P_{3} / \tan \theta_{1} \\
& \stackrel{(b)}{=} \xi\left(h_{11}+h_{12}\right)-\xi\left(h_{21}+h_{22}\right) h_{11} / h_{21} \\
& =\xi\left(h_{12} h_{21}-h_{11} h_{22}\right) / h_{21}=-\xi \operatorname{det}(\boldsymbol{H}) / h_{21},
\end{aligned}
$$

where step (a) follows from the fact that, $P P_{3}$ is equal to the co-ordinate of the point $D(\boldsymbol{H}, \xi)$ along the $u_{1}$-axis and therefore from (10), we have $P P_{3}=\xi\left(h_{11}+h_{12}\right)$. In step (a) we have also used the fact that since $O P_{1} P_{3}$ is a right angle triangle having $\angle O P_{3} P_{1}=\theta_{1}$. Hence, it follows that $P_{1} P_{3}=O P_{3} / \tan \theta_{1}$. Step (b) also follows from two facts. Firstly, $O P_{3}$ is equal to the co-ordinate of the point $D(\boldsymbol{H}, \xi)$ along the $u_{2}$-axis and therefore from (10), we have that $O P_{3}=$ $\xi\left(h_{21}+h_{22}\right)$ and secondly, from (19), we know that $\tan \theta_{1}=$ $h_{21} / h_{11}$. Similarly from Fig. 8 , we calculate the length of $P P_{2}$ as follows

$$
\begin{aligned}
P P_{2} & =P_{2} P_{3}-P P_{3} \\
& \stackrel{(a)}{=} O P_{3} / \tan \theta_{2}-\xi\left(h_{11}+h_{12}\right) \\
& \stackrel{(b)}{=} \xi\left(h_{21}+h_{22}\right) h_{12} / h_{22}-\xi\left(h_{11}+h_{12}\right) \\
& =\xi\left(h_{12} h_{21}-h_{11} h_{22}\right) / h_{22}=-\xi \operatorname{det}(\boldsymbol{H}) / h_{22},
\end{aligned}
$$

where step (a) follows from the fact that, $\mathrm{PP}_{3}$ is equal to the co-ordinate of the point $D(\boldsymbol{H}, \xi)$ along the $u_{1}$-axis and therefore from (10), we have that $P P_{3}=\xi\left(h_{11}+h_{12}\right)$. In step (a) we have also used the fact that $O P_{3} P_{2}$ is a right angle triangle having $\angle O P_{2} P_{3}=\theta_{2}$. Hence, it follows that $P_{2} P_{3}=O P_{3} / \tan \theta_{2}$. Step (b) follows from two facts. 
Firstly, $O P_{3}$ is equal to the co-ordinate of the point $D(\boldsymbol{H}, \xi)$ along the $u_{2}$-axis and therefore from (10), we have that $O P_{3}=\xi\left(h_{21}+h_{22}\right)$ and secondly, $\tan \theta_{2}=h_{22} / h_{12}$.

Using (43) and (44) in (42) we see that when $0 \leq \xi \leq$ $h_{22} /\left(h_{21}+h_{22}\right)=\min \left(h_{21}, h_{22}\right) /\left(h_{21}+h_{22}\right)$ (since $h_{21}>$ $h_{22}$ in scenario (a)), we have

$L_{1}^{\max }(\xi)=-2 \xi \operatorname{det}(\boldsymbol{H}) \min \left(\frac{1}{h_{21}}, \frac{1}{h_{22}}\right)=\frac{-2 \xi \operatorname{det}(\boldsymbol{H})}{\max \left(h_{22}, h_{21}\right)}$.

Computation of $L_{1}^{\max }(\xi)$ when $Q=D(\boldsymbol{H}, \xi) \in$ Region 2 : Point $Q=D(\boldsymbol{H}, \xi)$ lies in Region $2=C C^{\prime} A A^{\prime}$ if and only if

$$
O T \leq O Q \leq O M,
$$

where $M$ is the point of intersection of the line segment $A A^{\prime}$ and the diagonal $O B$ (see Fig. 8). In the following we firstly show that $D(\boldsymbol{H}, \xi) \in$ Region 2 if and only if

$$
\frac{h_{22}}{h_{21}+h_{22}} \leq \xi \leq \frac{h_{21}}{h_{21}+h_{22}} \text {. }
$$

Towards this end, we firstly derive an expression for $O M$. From the right angle triangle $O M_{1} M$ in Fig. 8, we know that $O M=M M_{1} / \sin \gamma$ and since $M M_{1}=h_{21}, \sin \gamma=$ $\left(h_{21}+h_{22}\right) / O B$. We have

$$
O M=\frac{h_{21} O B}{h_{21}+h_{22}} .
$$

Since the point $Q$ is nothing but the point $D(\boldsymbol{H}, \xi)$, from (10), we have $O Q=\xi O B$ and from (40), it follows that $O T=\frac{h_{22} O B}{h_{21}+h_{22}}$. In (46), we substitute $O Q$ by $\xi O B, O M$ by the R.H.S in (48) and $O T$ by $\frac{h_{22} O B}{h_{21}+h_{22}}$ to get

$$
\begin{array}{r}
\frac{h_{22} O B}{\left(h_{21}+h_{22}\right)} \leq \xi O B \leq \frac{h_{21} O B}{\left(h_{21}+h_{22}\right)} \\
\frac{h_{22}}{\left(h_{21}+h_{22}\right)} \leq \xi \leq \frac{h_{21}}{\left(h_{21}+h_{22}\right)}
\end{array}
$$

For all such values of the dimming target, $\xi$, satisfying (49), it follows that $D(\boldsymbol{H}, \xi) \in$ Region 2 . Next, we evaluate $L_{1}^{\max }(\xi)$ when $h_{22} /\left(h_{21}+h_{22}\right) \leq \xi \leq h_{21} /\left(h_{21}+h_{22}\right)$. For this scenario, from Fig. 8 we see that

$$
L_{1}^{\max }(\xi)=2 \min \left(Q Q_{1}, Q Q_{2}\right),
$$

where construction of $Q Q_{1}$ and $Q Q_{2}$ is similar to the construction of $P P_{1}$ and $P P_{2}$ (see Fig. 8), except the fact that $Q_{2}$ lies on $C B$ instead of $O C$. Next, we evaluate $Q Q_{1}$ and $Q Q_{2}$. To this end, using the similar steps as for the evaluation of $P P_{1}$ (see (43)) we have,

$$
Q Q_{1}=Q Q_{3}-Q_{1} Q_{3}=\frac{-\xi \operatorname{det}(\boldsymbol{H})}{h_{21}} .
$$

However, evaluation of $Q Q_{2}$ is not the same as evaluation of $P_{2}$, as the point $Q_{2}$ lies on the line segment $C B$ whereas the point $P_{2}$ lies on the line $O C$. Towards this end, using Fig. 8, we evaluate $Q Q_{2}$ as follows

$$
\begin{gathered}
Q Q_{2}=Q_{3} Q_{2}-Q_{3} Q \\
=Q_{3} Q_{4}+Q_{4} Q_{2}-Q_{3} Q \\
=h_{12}+\frac{C Q_{4}}{\tan \theta_{1}}-\xi\left(h_{11}+h_{12}\right) \\
=h_{12}+\frac{\xi\left(h_{21}+h_{22}\right)-h_{22}}{\left(h_{21} / h_{11}\right)}-\xi\left(h_{11}+h_{12}\right) \\
=\frac{-\operatorname{det}(\boldsymbol{H})(1-\xi)}{h_{21}}
\end{gathered}
$$

Using (51) and (52) in (50), we see that when $h_{22} /\left(h_{21}+\right.$ $\left.h_{22}\right) \leq \xi \leq h_{21} /\left(h_{21}+h_{22}\right)$, i.e. $\min \left(h_{21}, h_{22}\right) /\left(h_{21}+h_{22}\right) \leq$ $\xi \leq \max \left(h_{21}, h_{22}\right) /\left(h_{21}+h_{22}\right)$ (since $h_{21}>h_{22}$ in scenario (a)), we have

$$
\begin{gathered}
L_{1}^{\max }(\xi)=\frac{-2 \operatorname{det}(\boldsymbol{H}) \min (\xi,(1-\xi))}{h_{21}} \\
\stackrel{(a)}{=}-2 \operatorname{det}(\boldsymbol{H}) \frac{\min (\xi,(1-\xi))}{\max \left(h_{21}, h_{22}\right)},
\end{gathered}
$$

where step (a) follows from the fact that $h_{21}=\max \left(h_{21}, h_{22}\right)$, since for scenario (a), we know that $h_{21}>h_{22}$.

Computation of $L_{1}^{\max }(\xi)$ when $S=D(\boldsymbol{H}, \xi) \in$ Region 3:

Point $S=D(\boldsymbol{H}, \xi)$ lies in Region $3=A A^{\prime} B$ if and only if $O M \leq O S \leq O B$. Using (48) $\left(\frac{O M}{O B}=\frac{h_{21}}{h_{21}+h_{22}}\right)$ and the fact that $O S=\xi O B$ (from (10)), we have

$$
h_{21} /\left(h_{21}+h_{22}\right) \leq \xi \leq 1 .
$$

Next, we evaluate $L_{1}^{\max }(\xi)$ when $h_{21} /\left(h_{21}+h_{22}\right) \leq \xi \leq 1$. From Fig. 8 it is clear that when $S=D(\boldsymbol{H}, \xi) \in$ Region 3 then $L_{1}^{\max }(\xi)$ is given by

$$
L_{1}^{\max }(\xi)=2 \min \left(S S_{1}, S S_{2}\right),
$$

where $S_{1}$ and $S_{2}$ are the intersections of the straight line parallel to the $u_{1}$ axis passing through $S$, with the line segment $A B$ and $C B$ respectively. Next, we evaluate $S S_{1}$ and $S S_{2}$. To this end, using similar steps as for the evaluation of $Q Q_{2}$ (see (52)) we have,

$$
\begin{aligned}
S S_{2} & =S_{3} S_{2}-S_{3} S \\
& =S_{3} S_{4}+S_{4} S_{2}-S_{3} S \\
& =\frac{-\operatorname{det}(\boldsymbol{H})(1-\xi)}{h_{21}}
\end{aligned}
$$

However, evaluation of $S S_{1}$ is not same as evaluation of $Q Q_{1}$, as the point $S_{1}$ lies on the line segment $A B$ whereas the point $Q_{1}$ lies on the line $O A$. Towards the evaluation of $S S_{1}$, using Fig. 8, we have

$$
\begin{aligned}
S S_{1} & =S S_{3}-S_{1} S_{3} \\
& =\xi\left(h_{11}+h_{12}\right)-\left(S_{3} A_{1}+A_{1} S_{1}\right) \\
& =\xi\left(h_{11}+h_{12}\right)-\left(h_{11}+\frac{A A_{1}}{\tan \theta_{2}}\right) \\
& =\xi\left(h_{11}+h_{12}\right)-\left(h_{11}+\frac{\xi\left(h_{21}+h_{22}\right)-h_{21}}{\left(h_{22} / h_{12}\right)}\right) \\
& =\frac{-\operatorname{det}(\boldsymbol{H})(1-\xi)}{h_{22}}
\end{aligned}
$$

Using (57) and (58) in (56), we see that when 
$h_{21} /\left(h_{21}+h_{22}\right) \leq \xi \leq 1$, i.e. $\max \left(h_{21}, h_{22}\right) /\left(h_{21}+h_{22}\right) \leq$ $\xi \leq 1$ (since $h_{21}>h_{22}$ in scenario (a)), we have

$$
\begin{aligned}
L_{1}^{\max }(\xi) & =-2 \operatorname{det}(\boldsymbol{H})(1-\xi) \min \left(\frac{1}{h_{21}}, \frac{1}{h_{22}}\right) \\
& \stackrel{(a)}{=} \frac{-2 \operatorname{det}(\boldsymbol{H})(1-\xi)}{\max \left(h_{21}, h_{22}\right)},
\end{aligned}
$$

where step (a) follows from the fact that $h_{21}=\max \left(h_{21}, h_{22}\right)$, since for scenario (a), we know that $h_{21}>h_{22}$.

Therefore for the scenario (a), we have the expression of $L_{1}^{\max }(\xi)$ as follows

$$
L_{1}^{\max }(\xi)= \begin{cases}\frac{-2 \xi \operatorname{det}(\boldsymbol{H})}{\max \left(h_{21}, h_{22}\right)}, & 0 \leq \xi \leq \eta_{1} \triangleq \frac{\min \left(h_{21}, h_{22}\right)}{\left(h_{21}+h_{22}\right)} \\ \frac{-2 \operatorname{det}(\boldsymbol{H}) \min (\xi,(1-\xi))}{\max \left(h_{21}, h_{22}\right)}, & \eta_{1} \leq \xi \leq \eta_{2} \triangleq \frac{\max \left(h_{21}, h_{22}\right)}{\left(h_{21}+h_{22}\right)} \\ \frac{-2(1-\xi) \operatorname{det}(\boldsymbol{H})}{\max \left(h_{21}, h_{22}\right)}, & \eta_{2} \leq \xi \leq 1 .\end{cases}
$$

Since

$$
\eta_{1} \triangleq \frac{\min \left(h_{21}, h_{22}\right)}{\left(h_{21}+h_{22}\right)} \leq 1 / 2
$$

and

$$
\eta_{2} \triangleq \frac{\max \left(h_{21}, h_{22}\right)}{\left(h_{21}+h_{22}\right)} \geq 1 / 2,
$$

where the above two inequalities follows from the simple mathematical manipulations, and therefore the expression in (60) can be further simplified. To this end, we consider two cases based on the values of $\xi$.

Case (a): $0 \leq \xi \leq 1 / 2$

For this case we know that $\xi \leq(1-\xi)$ and hence

$$
\min (\xi,(1-\xi))=\xi
$$

Since from (61) and (62), we know that $\eta_{1} \leq 1 / 2$ and $\eta_{2} \geq$ $1 / 2$ and hence, for $0 \leq \xi \leq 1 / 2$ from (60) and (63) we have

$$
L_{1}^{\max }(\xi)=\frac{-2 \xi \operatorname{det}(\boldsymbol{H})}{\max \left(h_{21}, h_{22}\right)}
$$

Case (b): $1 / 2 \leq \xi \leq 1$

For this case we know that $\xi \geq(1-\xi)$ and hence

$$
\min (\xi,(1-\xi))=(1-\xi)
$$

Since from (61) and (62), we know that $\eta_{1} \leq 1 / 2$ and $\eta_{2} \geq$ $1 / 2$ and hence, for $1 / 2 \leq \xi \leq 1$ from (60) and (65) we have

$$
L_{1}^{\max }(\xi)=\frac{-2(1-\xi) \operatorname{det}(\boldsymbol{H})}{\max \left(h_{21}, h_{22}\right)} .
$$

Therefore from (64) and (66) the final expression of $L_{1}^{\max }(\xi)$ for scenario (a) is as follows

$$
L_{1}^{\max }(\xi)= \begin{cases}\frac{-2 \xi \operatorname{det}(\boldsymbol{H})}{\max \left(h_{21}, h_{22}\right)}, & 0 \leq \xi \leq 1 / 2 \\ \frac{-2(1-\xi) \operatorname{det}(\boldsymbol{H})}{\max \left(h_{21}, h_{22}\right)}, & 1 / 2 \leq \xi \leq 1\end{cases}
$$

Using similar arguments as for scenario (a), we evaluate $L_{1}^{\max }(\xi)$ for Scenario (b): $\left(h_{21} \leq h_{22}\right)$; and for Scenario (c): $\left(h_{12} \leq h_{11}\right.$ and $\left.h_{21}>h_{22}\right)$ as follows.

To this end, we first partition $R_{/ /}(\boldsymbol{H})$ into three regions as shown in Fig. 9. Next, we denote $D(\boldsymbol{H}, \xi)$ by the point $P$ if
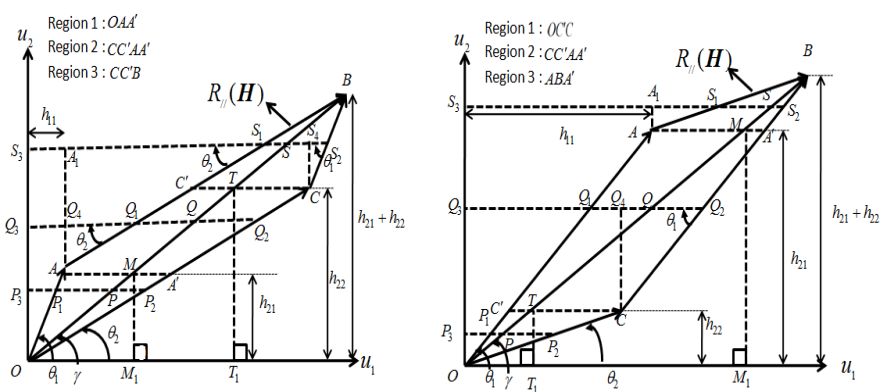

Fig. 9: Partition of the parallelogram $\mathrm{OABC} \triangleq R_{/ /}(\boldsymbol{H})$ into three different regions for the scenario (b) $\left(h_{21} \leq h_{22}\right)$; and scenario (c) $\left(h_{12} \leq h_{11}\right.$ and $\left.h_{21}>h_{22}\right)$ (left to right).

$D(\boldsymbol{H}, \xi)$ lies in Region 1, by the point $Q$ if $D(\boldsymbol{H}, \xi)$ lies in Region 2 and by the point $S$ if $D(\boldsymbol{H}, \xi)$ lies in Region 3.

Using (48) and (40) we can also show that the point $D(\boldsymbol{H}, \xi)$ lies in Region 1 if and only if $0 \leq \xi \leq \frac{\min \left(h_{21}, h_{22}\right)}{h_{22}+h_{22}}$, $D(\boldsymbol{H}, \xi)$ lies in Region 2 iff $\frac{\min \left(h_{21}, h_{22}\right)}{h_{21}+h_{22}} \leq \xi \leq \frac{\max \left(h_{21} h_{22}\right)}{h_{21}+h_{22}}$ and it lies in Region 3 iff $\frac{\max \left(h_{21}, h_{22}\right)}{h_{21}+h_{22}} \leq \xi \leq 1$. Next, we evaluate $L_{1}^{\max }(\xi)$ when $D(\boldsymbol{H}, \xi)$ lies in Region $i, i=1,2,3$.

Following similar steps as for scenario (a), from Fig. 9 it follows that when $D(\boldsymbol{H}, \xi) \in$ Region 1, i.e., when $0 \leq \xi \leq$ $\frac{\min \left(h_{21}, h_{22}\right)}{\left(h_{21}+h_{22}\right)}$

$$
L_{1}^{\max }(\xi)=\frac{-2 \xi \operatorname{det}(\boldsymbol{H})}{\max \left(h_{21}, h_{22}\right)}
$$

Similarly, using Fig. 9 it can be shown that when $D(\boldsymbol{H}, \xi) \in$ Region 2, i.e., when $\frac{\min \left(h_{21}, h_{22}\right)}{\left(h_{21}+h_{22}\right)} \leq \xi \leq \frac{\max \left(h_{21}, h_{22}\right)}{\left(h_{21}+h_{22}\right)}$

$$
L_{1}^{\max }(\xi)=-2 \operatorname{det}(\boldsymbol{H}) \frac{\min (\xi,(1-\xi))}{\max \left(h_{21}, h_{22}\right)} .
$$

Further, using Fig. 9 it can be shown that when $D(\boldsymbol{H}, \xi) \in$ Region 3, i.e., when $\frac{\max \left(h_{21}, h_{22}\right)}{\left(h_{21}+h_{22}\right)} \leq \xi \leq 1$

$$
L_{1}^{\max }(\xi)=\frac{-2(1-\xi) \operatorname{det}(\boldsymbol{H})}{\max \left(h_{21}, h_{22}\right)} .
$$

Following the steps used to arrive at (67) from (60) in scenario(a), for scenario (b) and (c) also we get the same final expression for $L_{1}^{\max }(\xi)$ as in (67). This completes the proof.

\section{APPENDIX B}

PROOF OF PROPOSITION 2

Proof:

Similar to proposition 1 , for proving proposition 2 , we consider three mutually exclusive scenarios. (a) $\left(h_{11}<h_{12}\right.$ and $\left.h_{21}>h_{22}\right)$; (b) $\left(h_{21} \leq h_{22}\right)$; and (c) $\left(h_{12} \leq h_{11}\right.$ and $\left.h_{21}>h_{22}\right)$. Moreover, from the definition of LED 1 and LED 2, it follows that, the channel matrix satisfies (20), i.e. $\operatorname{det}(\boldsymbol{H})<0$. For a fixed $\left(\boldsymbol{H}, P_{0} / \sigma, \xi\right)$, in the following, for a given $L_{1}=x, 0 \leq x \leq L_{1}^{\max }(\xi)$, we derive the expression for the maximum $L_{2}$, (i.e., length of the side of the rectangle along the $u_{2}$-axis (vertical length)), such that there exists a 


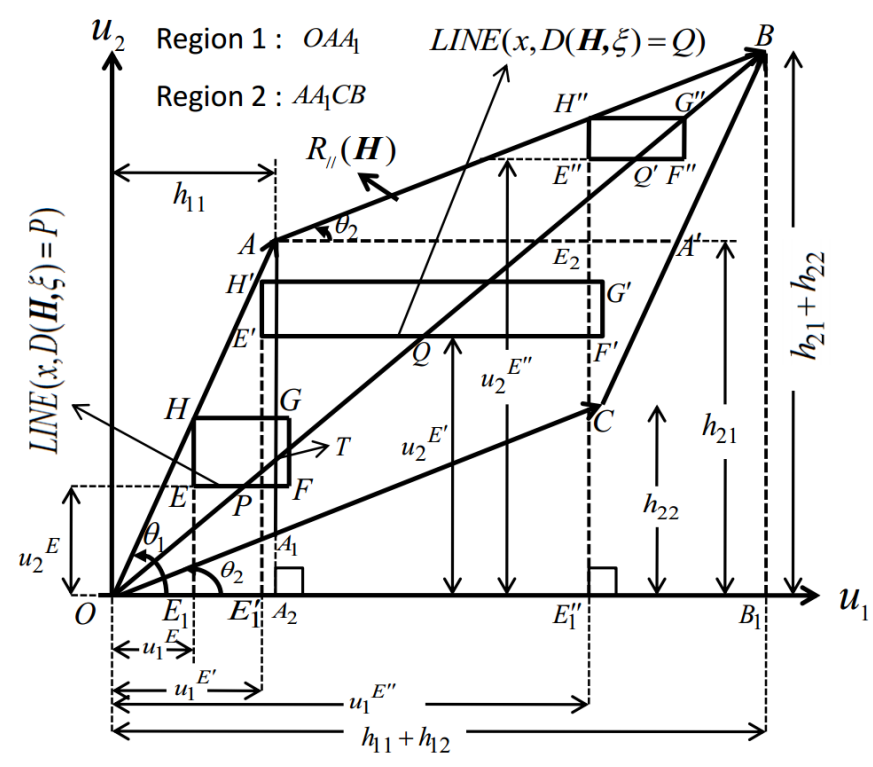

Fig. 10: Evaluation of $L_{2}^{u p, \xi}(x)$ for Scenario (a) $\left(h_{11}<\right.$ $h_{12}$ and $\left.h_{21}>h_{22}\right)$.

rectangle $\operatorname{Rect}\left(L_{1}=x, L_{2}, D(\boldsymbol{H}, \xi)\right) \subset R_{/ /}(\boldsymbol{H})$, i.e.

$$
L_{2}^{\xi}(x) \triangleq \max _{\substack{L_{2} \geq 0 \\ \operatorname{Rect}\left(x, L_{2}, D(\boldsymbol{H}, \xi)\right) \subset \mathcal{R} / /(\boldsymbol{H})}} L_{2} .
$$

To this end, for a fixed $\xi,^{13}$ and given $L_{1}=x$, we construct all such possible rectangles $\operatorname{Rect}\left(L_{1}=x, L_{2}, D(\boldsymbol{H}, \xi)\right) \subset$ $R_{/ /}(\boldsymbol{H})$ and among them we choose the rectangle having the maximum possible vertical length.

To get this rectangle we first construct a horizontal line segment of the length $x$ parallel to the $u_{1}$-axis such that its midpoint coincides with the point $D(\boldsymbol{H}, \xi)$. We denote this line segment by $\operatorname{LINE}(x, D(\boldsymbol{H}, \xi))$, i.e.

$\operatorname{LINE}(x, D(\boldsymbol{H}, \xi)) \triangleq\left\{v=\left(v_{1}, v_{2}\right) \in \mathbb{R}^{2} \mid v_{1} \in S_{1}, v_{2} \in S_{2}\right\}$,

where $S_{1} \triangleq\left\{v_{1} \in \mathbb{R}|| v_{1}-\xi\left(h_{11}+h_{12}\right) \mid \leq(x / 2)\right.$ and $S_{2} \triangleq\left\{v_{2} \in \mathbb{R} \mid v_{2}=\xi\left(h_{21}+h_{22}\right)\right\}$. Since $x \leq L_{1}^{\max }(\xi)$, from the definition of $L_{1}^{\max }(\xi)$ it follows that $\operatorname{LINE}(x, D(\boldsymbol{H}, \xi))$ lies completely inside $R_{/ /}(\boldsymbol{H})$.

Given any horizontal line segment of length $x$ parallel to the $u_{1}$-axis, any rectangle inside $R_{/ /}(\boldsymbol{H})$ having this line segment as one of its side can be constructed in two possible ways, either by extending it vertically downwards or extending it vertically upwards. ${ }^{14}$ Subsequently, we shall refer to these construction methods as "downward extension" and "upward extension".

Using this for a given $L_{2}>0$ we can construct a rectangle by extending the line segment $\operatorname{LINE}(x, D(\boldsymbol{H}, \xi))$ vertically

\footnotetext{
${ }^{13}$ Once we fix $\xi$, location of the point $D(\boldsymbol{H}, \xi)$ gets fixed (see (10)).

${ }^{14}$ By extending a horizontal line segment vertically downwards/upwards, we mean that we create a rectangle by drawing two vertical lines from the end points of this horizontal line segment in the downward/upward direction and then connecting the other two end points of these two vertical lines to form a rectangle.
}

upwards. We denote this rectangle by

$$
\operatorname{Rect}^{u p}\left(x, L_{2}, D(\boldsymbol{H}, \xi)\right) \triangleq \operatorname{Rect}\left(x, L_{2}, D(\boldsymbol{H}, \xi)+C_{0}\right),
$$

where $C_{0} \triangleq\left(0, L_{2} / 2\right)$. Let $L_{2}^{u p, \xi}(x)$ denote the largest possible vertical length of all such rectangles which lie completely inside $R_{/ /}(\boldsymbol{H})$ and are constructed by the upward extension of the line segment $\operatorname{LINE}(x, D(\boldsymbol{H}, \xi))$, i.e.

$$
L_{2}^{u p, \xi}(x) \triangleq \max _{\operatorname{Rect}_{2} \geq 0} L_{2} .
$$

Similarly, we construct any rectangle of vertical length $L_{2} \geq 0$ by extending the line segment $\operatorname{LINE}(x, D(\boldsymbol{H}, \xi))$ vertically downwards. We denote this rectangle by

$$
\operatorname{Rect}^{\text {down }}\left(x, L_{2}, D(\boldsymbol{H}, \xi)\right) \triangleq \operatorname{Rect}\left(x, L_{2}, D(\boldsymbol{H}, \xi)+C_{1}\right),
$$

where $C_{1} \triangleq\left(0,-L_{2} / 2\right)$. Let $L_{2}^{\text {down, } \xi}(x)$ denote the largest possible vertical length of all such rectangles which lie completely inside $R_{/ /}(\boldsymbol{H})$ and are constructed using the "downward extension" method, i.e.

$$
L_{2}^{\text {down }, \xi}(x) \triangleq \max _{\substack{L_{2} \geq 0 \\ \operatorname{Rect}^{\text {down }}\left(x, L_{2}, D(\boldsymbol{H}, \xi)\right) \subset R_{/ /}(\boldsymbol{H})}} L_{2} .
$$

$L_{2}^{\xi}(x)$ is the maximum possible vertical length of any rectangle lying completely inside $R_{/ /}(\boldsymbol{H})$ with horizontal side length equal to $x$ and having its mid point at $D(\boldsymbol{H}, \xi)$, which is the midpoint of the line segment $\operatorname{LINE}(x, D(\boldsymbol{H}, \xi))$. Equivalently, such a maximal rectangle ${ }^{15}$ must be symmetric about the line segment $\operatorname{LINE}(x, D(\boldsymbol{H}, \xi))$. Since such a maximal rectangle $\operatorname{Rect}\left(x, L_{2}^{\xi}(x), D(\boldsymbol{H}, \xi)\right)$ lies inside $R_{/ /}(\boldsymbol{H})$, from (74) and (76) it follows that

$$
\operatorname{Rect}\left(x, L_{2}^{\xi}(x), D(\boldsymbol{H}, \xi)\right) \subset S_{3} \cup S_{4},
$$

where $S_{3} \triangleq \operatorname{Rect}^{u p}\left(x, L_{2}^{u p, \xi}(x), D(\boldsymbol{H}, \xi)\right) \quad$ and $S_{4} \triangleq \operatorname{Rect}^{\text {down }}\left(x, L_{2}^{\text {down }, \xi}(x), D(\boldsymbol{H}, \xi)\right) \quad$ and $S_{3} \cap S_{4}=\operatorname{LINE}(x, D(\boldsymbol{H}, \xi))$. Further since the maximal rectangle $\operatorname{Rect}\left(x, L_{2}^{\xi}(x), D(\boldsymbol{H}, \xi)\right)$ has the maximum possible vertical length and is symmetric about $\operatorname{LINE}(x, D(\boldsymbol{H}, \xi))$, it follows that $L_{2}^{\xi}(x) / 2=L_{2}^{u p, \xi}(x)$ if $L_{2}^{u p, \xi}(x) \leq L_{2}^{\text {down, }, \text {, }}$ and $L_{2}^{\xi}(x) / 2=L_{2}^{\text {down, } \xi}(x)$ if $L_{2}^{\text {down, } \xi}(x) \leq L_{2}^{u p, \xi}(x)$, i.e.

$$
L_{2}^{\xi}(x)=2 \min \left(L_{2}^{\text {down }, \xi}(x), L_{2}^{u p, \xi}(x)\right) .
$$

We next derive expressions for $L_{2}^{u p, \xi}(x)$ and $L_{2}^{\text {down, } \xi}(x)$ for $0 \leq x \leq L_{1}^{\max }(\xi)$ for a fixed $0 \leq \xi \leq 1$. We firstly consider scenario (a) $\left(h_{11}<h_{12}\right.$ and $\left.h_{21}>h_{22}\right)$.

\section{A. Computation of $L_{2}^{u p, \xi}(x)$ for scenario (a)}

Towards this end, we divide $R_{/ /}(\boldsymbol{H})$ into two regions, Region $i, i=1,2$, namely Region $1=O A A_{1}$ and Region 2 $=A A_{1} C B$ (see Fig. 10). Note that in Fig. 10, the straight line $A A_{1} A_{2}$ is parallel to the $u_{2}$-axis and $A_{1}$ is the point of intersection of this line segment with the side OC of $R_{/ /}(\boldsymbol{H})$. Next, we evaluate expressions for $L_{2}^{u p, \xi}(x)$ depending upon

\footnotetext{
15 By the maximal rectangle we mean, the rectangle with the maximum possible vertical length for a given horizontal length and which lies completely inside the rectangle $R_{/ /}(\boldsymbol{H})$.
} 
the region where $D(\boldsymbol{H}, \xi)$ lies. In Fig. 10, we denote $D(\boldsymbol{H}, \xi)$ by the point $P$ if $D(\boldsymbol{H}, \xi)$ lies in Region 1 and by the point $Q / Q^{\prime}$ if $D(\boldsymbol{H}, \xi)$ lies in Region 2.

Computation of $L_{2}^{u p, \xi}(x)$ when $D(\boldsymbol{H}, \xi)=P \in$ Region 1: The point $D(\boldsymbol{H}, \xi)=P \in$ Region $1=O A A_{1}$ iff

$$
0 \leq O P \leq O T,
$$

where $T$ is the point of intersection of the line segment $A A_{2}$ with the diagonal $O B$ (see Fig. 10). Next, we evaluate expression for $O T$. Towards this end, from the similarity of the triangles $O T A_{2}$ and $O B B_{1}$ it follows that $\frac{O T}{O B}=\frac{O A_{2}}{O B_{1}}$. Further, from Fig. 10, it follows that $O A_{2}=h_{11}$ and $O B_{1}=h_{11}+h_{12}$ and therefore we have,

$$
O T=\frac{h_{11}}{h_{11}+h_{12}} O B .
$$

Since the point $P$ is nothing but the point $D(\boldsymbol{H}, \xi)$, from (10) we have $O P=\xi O B$. Therefore, using (79) and $O P=\xi O B$ in (78) we have, $D(\boldsymbol{H}, \xi) \in$ Region 1 iff $0 \leq \xi \leq \frac{h_{11}}{h_{11}+h_{12}}$.

When $0 \leq \xi \leq \frac{h_{11}}{h_{11}+h_{12}}$, from (74) it follows that for evaluating $L_{2}^{u p, \xi}(x)$, we need to construct rectangles $\operatorname{Rect}^{u p}\left(x, L_{2}, D(\boldsymbol{H}, \xi)\right)$ using the "upward extension" of the line segment $\operatorname{LINE}(x, D(\boldsymbol{H}, \xi)=P)=E F$ as shown in Fig. 10. $L_{2}^{u p, \xi}(x)$ is then the largest possible vertical length of all such rectangles which lie inside $R_{/ /}(\boldsymbol{H})$. From Fig. 10, it is clear that during the upward extension of the line $E F$, with increasing vertical length $L_{2}$ of the constructed rectangle $\operatorname{Rect}^{u p}\left(x, L_{2}, D(\boldsymbol{H}, \xi)\right)$, the vertically upward line from $E$ will be the first to move out of $R_{/ /}(\boldsymbol{H})$ when compared to the vertical line from $F$. Hence it follows that in Fig. 10, for $x=E F$ and $0 \leq \xi \leq \frac{h_{11}}{h_{11}+h_{12}}$, we have $L_{2}^{u p, \xi}(x)=E H$. To evaluate $E H$, we firstly denote the $\left(u_{1}, u_{2}\right)$ coordinates of the point $E$ by $\left(u_{1}^{E}, u_{2}^{E}\right)$. From the definition of $\operatorname{LINE}(x, D(\boldsymbol{H}, \xi)$ and (10) it is clear that

$$
u_{1}^{E}=\xi\left(h_{11}+h_{12}\right)-x / 2, \quad u_{2}^{E}=\xi\left(h_{21}+h_{22}\right) .
$$

When $0 \leq \xi \leq \frac{h_{11}}{h_{11}+h_{12}}$ and $0 \leq x \leq L_{1}^{\max }(\xi)$, using Fig. 10, $E H$ is computed as follows

$$
\begin{aligned}
L_{2}^{u p, \xi}(x)=E H & =E_{1} H-E_{1} E \\
& =u_{1}^{E} \tan \theta_{1}-u_{2}^{E} \\
& \stackrel{(a)}{=}\left(\xi\left(h_{11}+h_{12}\right)-\frac{x}{2}\right) \frac{h_{21}}{h_{11}}-\xi\left(h_{21}+h_{22}\right) \\
& =\frac{-\xi \operatorname{det}(\boldsymbol{H})-\frac{x}{2} h_{21}}{h_{11}}
\end{aligned}
$$

where $E_{1}$ is the point of intersection of the extension of the line segment $E H$ and the $u_{1}$-axis (see Fig. 10). Step (a) follows from (80) and (19).

Computation of $L_{2}^{u p, \xi}(x)$ when $D(\boldsymbol{H}, \xi)=Q \in$ Region 2: Point $D(\boldsymbol{H}, \xi)=Q$ lies in Region $2=A A_{1} C B$ if and only if $O T \leq O Q \leq O B$. Since $Q$ denote the point $D(\boldsymbol{H}, \xi)$, from (10) we have, $O Q=\xi O B$ and from (79) we have $O T=\frac{h_{11} O B}{h_{11}+h_{12}}$. Hence, it follows that $D(\boldsymbol{H}, \xi)$ lies in Region 2 iff $\frac{h_{11}}{h_{11}+h_{12}} \leq \xi \leq 1$. We next evaluate $L_{2}^{u p, \xi}(x)$ when $\xi$ lies in this interval.

From (74), it follows that for evaluating $L_{2}^{u p, \xi}(x)$, we need to construct rectangles $\operatorname{Rect}^{u p}\left(x, L_{2}, Q=D(\boldsymbol{H}, \xi)\right)$ using the "upward extension" of the line segment $\operatorname{LINE}(x, Q=$ $D(\boldsymbol{H}, \xi))$ as shown in Fig. 10. $L_{2}^{u p, \xi}(x)$ is then the largest possible vertical length of all such rectangles which lie inside $R_{/ /}(\boldsymbol{H})$. From Fig. 10, it is clear that during the upward extension of the line segment $\operatorname{LINE}(x, Q=D(\boldsymbol{H}, \xi))$, the upper left vertex of the constructed rectangle having the largest vertical length will either intersect with the side $O A$ of $R_{/ /}(\boldsymbol{H})$ or with the side $A B$ of $R_{/ /}(\boldsymbol{H})$ (see rectangles $E^{\prime} F^{\prime} G^{\prime} H^{\prime}$ and $E^{\prime \prime} F^{\prime \prime} G^{\prime \prime} H^{\prime \prime}$ in Fig. 10). The upper left vertex intersects with the side $O A$ if and only if the lower left vertex of the constructed rectangle, (i.e., the leftmost point of the line segment $\operatorname{LINE}(x, D(\boldsymbol{H}, \xi))$ (see $E^{\prime}$ in Fig. 10) lies inside Region 1, i.e.

$$
\begin{gathered}
u_{1}^{D(\boldsymbol{H}, \xi)}-x / 2 \leq h_{11}, \text { i.e. } \\
2 \xi h_{12}-2(1-\xi) h_{11} \leq x,
\end{gathered}
$$

where $u_{1}$ coordinate of the point $D(\boldsymbol{H}, \xi)$ is denoted by $u_{1}^{D(\boldsymbol{H}, \xi)}$. From (10), we know that $u_{1}^{D(\boldsymbol{H}, \xi)}=\xi\left(h_{11}+h_{12}\right)$. On the other hand, the upper left vertex intersects with the side $A B$ of $R_{/ /}(\boldsymbol{H})$ if and only if the lower left vertex of the constructed rectangle, i.e., the leftmost point of the line segment $\operatorname{LINE}\left(x, Q^{\prime}=D(\boldsymbol{H}, \xi)\right)$ (see $E^{\prime \prime}$ in Fig. 10) lies inside Region 2, i.e.

$$
\begin{gathered}
u_{1}^{D(\boldsymbol{H}, \xi)}-x / 2 \geq h_{11}, \text { i.e. } \\
x \leq 2 \xi h_{12}-2(1-\xi) h_{11} .
\end{gathered}
$$

From the above, we know that when $x$ satisfies (82), i.e. $2 \xi h_{12}-2(1-\xi) h_{11} \leq x$, the lower left vertex of the constructed rectangle lies in Region 1 and the upper left vertex lies on he side $O A$. Hence, we have

$$
\begin{aligned}
L_{2}^{u p, \xi}(x) & =E^{\prime} H^{\prime}=E_{1}^{\prime} H^{\prime}-E_{1}^{\prime} E^{\prime} \\
& =\frac{-\xi \operatorname{det}(\boldsymbol{H})-\frac{x}{2} h_{21}}{h_{11}}
\end{aligned}
$$

Similarly, when $x$ satisfies (83), i.e. $2 \xi h_{12}-2(1-\xi) h_{11} \geq$ $x$, the lower left vertex of the constructed rectangle lies in Region 2 and the upper left vertex lies on the side $A B$. Hence, we have

$$
\begin{aligned}
L_{2}^{u p, \xi}(x) & =E^{\prime \prime} H^{\prime \prime} \\
& =E_{1}^{\prime \prime} H^{\prime \prime}-E_{1}^{\prime \prime} E^{\prime \prime} \\
& =E_{1}^{\prime \prime} E_{2}+E_{2} H^{\prime \prime}-E_{1}^{\prime \prime} E^{\prime \prime} \\
& \stackrel{(a)}{=} h_{21}+A E_{2} \tan \theta_{2}-E_{1}^{\prime \prime} E^{\prime \prime} \\
& \stackrel{(b)}{=} h_{21}+\left(u_{1}^{E^{\prime \prime}}-h_{11}\right) \tan \theta_{2}-u_{2}^{E^{\prime \prime}} \\
& \stackrel{(c)}{=} h_{21}+\left(\xi\left(h_{11}+h_{12}\right)-\frac{x}{2}-h_{11}\right) \frac{h_{22}}{h_{12}}-\xi\left(h_{21}+h_{22}\right) \\
& =\frac{-(1-\xi) \operatorname{det}(\boldsymbol{H})-\frac{x}{2} h_{22}}{h_{12}},
\end{aligned}
$$

where $E_{2}$ is the point of intersection of the line $E^{\prime \prime} H^{\prime \prime}$ with $A A^{\prime}$ and $\left(u_{1}^{E^{\prime \prime}}, u_{2}^{E^{\prime \prime}}\right)$ are the $\left(u_{1}, u_{2}\right)$ coordinates of the point $E^{\prime \prime}$. Step (a) follows from right angle triangle $A E_{2} H^{\prime \prime}$. Step (b) follows from the fact that, $A E_{2}=u_{1}^{E^{\prime \prime}}-h_{11}$ and $E_{1} E^{\prime \prime}=$ $u_{2}^{E^{\prime \prime}}$. In step (c) the expression for $u_{1}^{E^{\prime \prime}}$ and $u_{2}^{E^{\prime \prime}}$ follows from the definition of $\operatorname{LINE}(x, D(\boldsymbol{H}, \xi))$ in (72) and (10) and the value of $\tan \theta_{2}$ follows from (19). Therefore, when $D(\boldsymbol{H}, \xi) \in$ 


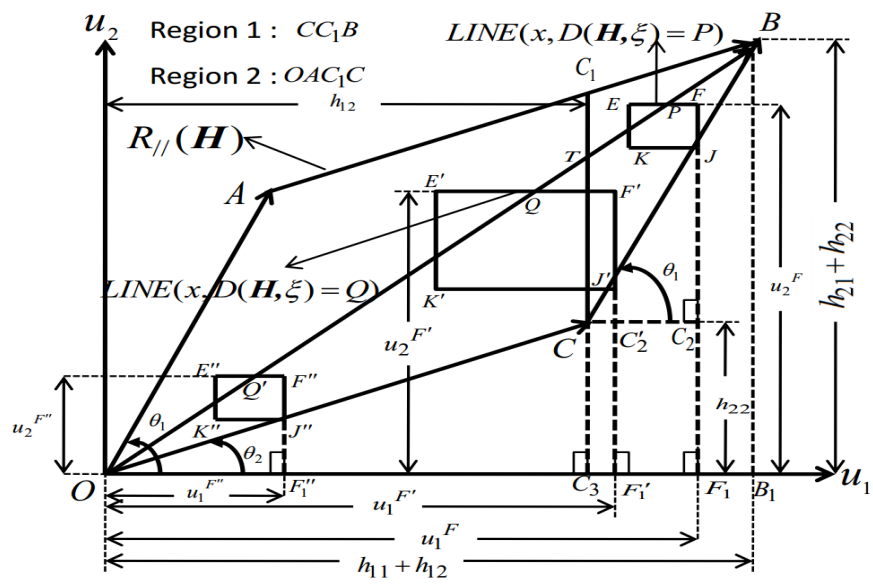

Fig. 11: Evaluation of $L_{2}^{\text {down, } \xi}(x)$ for Scenario (a) $\left(h_{11}<\right.$ $h_{12}$ and $\left.h_{21}>h_{22}\right)$.

Region 2, (i.e., $\left.\frac{h_{11}}{h_{11}+h_{12}} \leq \xi \leq 1\right)$ and $\left(0 \leq x \leq 2 \xi h_{12}-2(1-\right.$ $\xi) h_{11}$, we have

$$
L_{2}^{u p, \xi}(x)=\frac{-(1-\xi) \operatorname{det}(\boldsymbol{H})-\frac{x}{2} h_{22}}{h_{12}}
$$

Therefore, in scenario (a) $\left(h_{11}<h_{12}\right.$ and $\left.h_{21}>h_{22}\right)$, from (81), (84) and (85) we finally have

$L_{2}^{u p, \xi}(x)=\left\{\begin{array}{lc}\frac{-\xi \operatorname{det}(\boldsymbol{H})-\frac{x}{2} h_{21}}{h_{11}} & 0 \leq \xi \leq \mu_{1} \text { and } 0 \leq x \leq L_{1}^{\max }(\xi) \\ \frac{-(1-\xi) \operatorname{det}(\boldsymbol{H})-\frac{x}{2} h_{22}}{h_{12}} & \mu_{1} \leq \xi \leq 1 \text { and } 0 \leq x \leq \eta_{3}(\xi) \\ \frac{-\xi \operatorname{det}(\boldsymbol{H})-\frac{x}{2} h_{21}}{h_{11}} & \mu_{1} \leq \xi \leq 1 \text { and } \eta_{3}(\xi) \leq x \leq L_{1}^{\max }(\xi)\end{array}\right.$

where $\mu_{1} \triangleq \frac{h_{11}}{h_{11}+h_{12}}$ and $\eta_{3}(\xi) \triangleq 2 \xi h_{12}-2(1-\xi) h_{11}$. In the next section, we derive expressions for $L_{2}^{\text {down, } \xi}(x)$ for scenario (a).

\section{B. Computation of $L_{2}^{\text {down, } \xi}(x)$ for scenario (a)}

Evaluation of $L_{2}^{\text {down, } \xi}(x)$ is similar to that of $L_{2}^{u p, \xi}(x)$. In Fig. 11, we partition the parallelogram $R_{/ /}(\boldsymbol{H})$ into two regions, namely, Region $1=C C_{1} B$ and Region $2=O A C_{1} C$. Next, we evaluate the expression for $L_{2}^{\text {down }, \xi}(x)$ depending upon the region where $D(\boldsymbol{H}, \xi)$ lies. In Fig. 11, we denote $D(\boldsymbol{H}, \xi)$ by the point $P$ if $D(\boldsymbol{H}, \xi)$ lies in Region 1, by point $Q / Q^{\prime}$ if $D(\boldsymbol{H}, \xi)$ lies in Region 2.

Computation of $L_{2}^{\text {down, } \xi}(x)$ when $D(\boldsymbol{H}, \xi) \in$ Region 1:

The point $D(\boldsymbol{H}, \xi)=P \in$ Region $1=C C_{1} B$ iff

$$
O T \leq O P \leq O B,
$$

where $T$ is the point of intersection of the straight line $C C_{1}$ with the diagonal $O B$ (see Fig. 11). Note that the straight line $C C_{1}$ is parallel to the $u_{2}$ axis (see Fig. 11). Next, we derive an expression for $O T$. Towards this end, from the similarity of the triangles $O T C_{3}$ and $O B B_{1}$ it follows that

$$
O T=\frac{h_{12}}{h_{11}+h_{12}} O B .
$$

Since $P=D(\boldsymbol{H}, \xi)$, from (10) we have $O P=\xi O B$. Therefore, using (88) and $O P=\xi O B$ in (87) we have,
$D(\boldsymbol{H}, \xi) \in$ Region 1 iff $\frac{h_{12}}{h_{11}+h_{12}} \leq \xi \leq 1$.

When $\frac{h_{12}}{h_{11}+h_{12}} \leq \xi \leq 1$, from (76), it follows that for evaluating $L_{2}^{\text {down }, \xi}(x)$, we need to construct rectangles $\operatorname{Rect}^{\text {down }}\left(x, L_{2}, D(\boldsymbol{H}, \xi)\right)$ using the "downward extension" of the line segment $L I N E(x, D(\boldsymbol{H}, \xi)=P)=E F$ as shown in Fig. 11. $L_{2}^{\text {down, } \xi}(x)$ is then the largest possible vertical length of all such rectangles which lie inside $R_{/ /}(\boldsymbol{H})$. From Fig. 11, it is clear that during the downward extension of the line $E F$, with increasing vertical length $L_{2}$ of the constructed rectangle $\operatorname{Rect}^{\text {down }}\left(x, L_{2}, D(\boldsymbol{H}, \xi)\right)$, the vertically downward line from $F$ will be the first to move out of $R_{/ /}(\boldsymbol{H})$ when compared to the vertical line from $E$. Hence, it follows that in Fig. 11, for $x=E F$ and $\frac{h_{12}}{h_{11}+h_{12}} \leq \xi \leq 1$, we have $L_{2}^{\text {down, } \xi}(x)=F J$. Let us denote the $\left(u_{1}, u_{2}\right)$ coordinates of the point $F$ by $\left(u_{1}^{F}, u_{2}^{F}\right)$. From the definition of $\operatorname{LINE}(x, P=D(\boldsymbol{H}, \xi))$ in (72) and from (10) we have

$$
u_{1}^{F}=\xi\left(h_{11}+h_{12}\right)+\frac{x}{2}, \quad u_{2}^{F}=\xi\left(h_{21}+h_{22}\right) .
$$

When $\frac{h_{12}}{h_{11}+h_{12}} \leq \xi \leq 1$ and $0 \leq x \leq L_{1}^{\max }(\xi)$, using Fig. 11, $F J$ is computed as follows

$$
\begin{aligned}
L_{2}^{\text {down }, \xi}(x) & =F J \\
& =F_{1} F-F_{1} J \\
& =F_{1} F-F_{1} C_{2}-C_{2} J \\
& \stackrel{(a)}{=} F_{1} F-h_{22}-C C_{2} \tan \theta_{1} \\
& \stackrel{(b)}{=} u_{2}^{F}-h_{22}-\left(u_{1}^{F}-h_{12}\right) \tan \theta_{1} \\
& \stackrel{(c)}{=} \xi\left(h_{21}+h_{22}\right)-h_{22}-\left(\xi\left(h_{11}+h_{12}\right)+\frac{x}{2}-h_{12}\right) \frac{h_{21}}{h_{11}} \\
& =\frac{-(1-\xi) \operatorname{det}(\boldsymbol{H})-\frac{x}{2} h_{21}}{h_{11}},
\end{aligned}
$$

where $F_{1}$ is the point of intersection of the extension of the line $F J$ with the $u_{1}$-axis (see Fig. 11). Step (a) follows from the right angle triangle $C_{2} J$. Step (b) follows from the fact that, $C C_{2}=u_{1}^{F}-h_{12}$ and $F_{1} F=u_{2}^{F}$. In step (c) we use the expressions for $u_{1}^{F}$ and $u_{2}^{F}$ from (89) and the value of $\tan \theta_{1}$ from (19).

Computation of $L_{2}^{\text {down, } \xi}(x)$ when $Q=D(\boldsymbol{H}, \xi) \in$ Region 2 : We know from Fig. 11, that $Q=D(\boldsymbol{H}, \xi) \in$ Region $2=$ $O A C_{1} C$ iff

$$
0 \leq O Q \leq O T .
$$

Since $Q$ is nothing but $D(\boldsymbol{H}, \xi)$, from (10) we have $O Q=$ $\xi O B$. Further, from (88) we have $O T=\frac{h_{12}}{h_{11}+h_{12}} O B$. Therefore, it follows that $D(\boldsymbol{H}, \xi) \in$ Region 2 iff $0 \leq \xi \leq$ $h_{12} /\left(h_{11}+h_{12}\right)$. Next, we evaluate $L_{2}^{\text {down, } \xi}(x)$ when $\xi$ lies in this interval.

From (76), it follows that for evaluating $L_{2}^{\text {down, } \xi}(x)$, we need to construct rectangles $\operatorname{Rect}^{\text {down }}\left(x, L_{2}, D(\boldsymbol{H}, \xi)\right)$ using the "downward extension" of the line segment $\operatorname{LINE}(x, D(\boldsymbol{H}, \xi))$ as shown in Fig. 11. $L_{2}^{\text {down }, \xi}(x)$ is then the largest possible vertical length of all such rectangles which lie inside $R_{/ /}(\boldsymbol{H})$. From Fig. 11, it is clear that during the downward extension of the line segment $\operatorname{LINE}(x, D(\boldsymbol{H}, \xi))$, the lower right vertex of the constructed rectangle having the largest vertical length will either intersect with the side $O C$ of $R_{/ /}(\boldsymbol{H})$ or with the side $C B$ of $R_{/ /}(\boldsymbol{H})$ (see rectangles 
$E^{\prime} F^{\prime} J^{\prime} K^{\prime}$ and $E^{\prime \prime} F^{\prime \prime} J^{\prime \prime} K^{\prime \prime}$ in Fig. 11). The lower right vertex intersects with the side $C B$ if and only if the upper right vertex of the constructed rectangle, (i.e., the rightmost point of the line segment $\operatorname{LINE}(x, D(\boldsymbol{H}, \xi))$ (see $F^{\prime}$ in Fig. 11) lies inside Region 1, i.e.

$$
\begin{gathered}
u_{1}^{D(\boldsymbol{H}, \xi)}+x / 2 \geq h_{12}, \text { i.e. } \\
x \geq 2(1-\xi) h_{12}-2 \xi h_{11},
\end{gathered}
$$

where $u_{1}^{D(\boldsymbol{H}, \xi)}$ denote the $u_{1}$ coordinate of the point $D(\boldsymbol{H}, \xi)$. From (10) we know that $u_{1}^{D(\boldsymbol{H}, \xi)}=\xi\left(h_{11}+h_{12}\right)$. On the other hand, the lower right vertex of the constructed rectangle intersects with the side $O C$ of $R_{/ /}(\boldsymbol{H})$ if and only if the upper right vertex of the constructed rectangle (i.e., the rightmost point of the line segment $\operatorname{LINE}(x, D(\boldsymbol{H}, \xi))$ ) (see $F^{\prime \prime}$ in Fig. 11) lies inside Region 2, i.e.

$$
\begin{gathered}
u_{1}^{D(\boldsymbol{H}, \xi)}+x / 2 \leq h_{12}, \text { i.e. } \\
x \leq 2(1-\xi) h_{12}-2 \xi h_{11} .
\end{gathered}
$$

From the above, we know that when $x \leq 2(1-\xi) h_{12}-2 \xi h_{11}$, the upper right vertex of the constructed rectangle lies in Region 2 and the lower right vertex lies on the side $O C$. Hence, we have $L_{2}^{\text {down, } \xi}(x)=F^{\prime \prime} J^{\prime \prime}$. Towards this end, we firstly denote the $\left(u_{1}, u_{2}\right)$ coordinates of the point $F^{\prime \prime}$ by $\left(u_{1}^{F^{\prime \prime}}, u_{2}^{F^{\prime \prime}}\right)$. From the definition of $\operatorname{LINE}(x, D(\boldsymbol{H}, \xi))$ in (72) and from (10) we have

$$
u_{1}^{F^{\prime \prime}}=\xi\left(h_{11}+h_{12}\right)+\frac{x}{2}, \quad u_{2}^{F^{\prime \prime}}=\xi\left(h_{21}+h_{22}\right) .
$$

Next, for $0 \leq \xi \leq h_{12} /\left(h_{11}+h_{12}\right)$ and $0 \leq x \leq 2(1-\xi) h_{12}-$ $2 \xi h_{11}$, we evaluate expression for $L_{2}^{\text {down, } \xi}(x)=F^{\prime \prime} J^{\prime \prime}$ as follows.

$$
\begin{aligned}
L_{2}^{\text {down }, \xi}(x) & =F^{\prime \prime} J^{\prime \prime} \\
& =F_{1}^{\prime \prime} F^{\prime \prime}-F_{1}^{\prime \prime} J^{\prime \prime} \\
& \stackrel{(a)}{=} u_{2}^{F^{\prime \prime}}-u_{1}^{F^{\prime \prime}} \tan \theta_{2} \\
& \stackrel{(b)}{=} \xi\left(h_{21}+h_{22}\right)-\left(\xi\left(h_{11}+h_{12}\right)+\frac{x}{2}\right) \frac{h_{22}}{h_{12}} \\
& =\frac{-\xi \operatorname{det}(\boldsymbol{H})-\frac{x}{2} h_{22}}{h_{12}},
\end{aligned}
$$

where $F_{1}^{\prime \prime}$ is the point of intersection of the line $F^{\prime \prime} J^{\prime \prime}$ extended downward with the $u_{1}$-axis (see Fig. 11). Step (a) follows from the two facts. Firstly, from the fact that $F_{1}^{\prime \prime} F^{\prime \prime}$ is the $u_{2}$ coordinate $F^{\prime \prime}$, and secondly from the right angle triangle $O F_{1}^{\prime \prime} J^{\prime \prime}$, we have $\tan \theta_{2}=\frac{F_{1}^{\prime \prime} J^{\prime \prime}}{u_{1}^{F^{\prime \prime}}}$, i.e. $F_{1}^{\prime \prime} J^{\prime \prime}=u_{1}^{F^{\prime \prime}} \tan \theta_{2}$. Step (b) follows from (94) and (19).

On the other hand, when $x \geq 2(1-\xi) h_{12}-2 \xi h_{11}$, the upper right vertex of the constructed rectangle lies in Region 1 and the lower right vertex lies on the side $C B$. Hence, we have $L_{2}^{\text {down, } \xi}(x)=F^{\prime} J^{\prime}$. Towards this end, we firstly denote the $\left(u_{1}, u_{2}\right)$ coordinates of the point $F^{\prime}$ by $\left(u_{1}^{F^{\prime}}, u_{2}^{F^{\prime}}\right)$. From the definition of $\operatorname{LINE}(x, D(\boldsymbol{H}, \xi))$ in (72) and from (10) we have

$$
u_{1}^{F^{\prime}}=\xi\left(h_{11}+h_{12}\right)+\frac{x}{2}, \quad u_{2}^{F^{\prime}}=\xi\left(h_{21}+h_{22}\right) .
$$

The steps involved in the evaluation of $F^{\prime} J^{\prime}$ is exactly the same as for the evaluation of $F J$ in (90). Hence, from Fig. 11 we have

$$
\begin{aligned}
& \begin{array}{r}
L_{2}^{\text {down }, \xi}(x)=F^{\prime} J^{\prime} \\
=F_{1}^{\prime} F^{\prime}-F_{1}^{\prime} J^{\prime}
\end{array} \\
& =\frac{-(1-\xi) \operatorname{det}(\boldsymbol{H})-\frac{x}{2} h_{21}}{h_{11}},
\end{aligned}
$$

Therefore, in scenario (a) $\left(h_{11}<h_{12}\right.$ and $\left.h_{21}>h_{22}\right)$, from (90), (95) and (97) we finally have

$$
L_{2}^{\operatorname{down}, \xi}(x)= \begin{cases}\frac{-(1-\xi) \operatorname{det}(\boldsymbol{H})-\frac{x}{2} h_{21}}{h_{11}}, \mu_{2} \leq \xi \leq 1 \text { and } 0 \leq x \leq L_{1}^{\max }(\xi) \\ \frac{-\xi \operatorname{det}(\boldsymbol{H})-\frac{x}{2} h_{22}}{h_{12}}, & 0 \leq \xi \leq \mu_{2} \text { and } 0 \leq x \leq \eta_{4}(\xi) \\ \frac{-(1-\xi) \operatorname{det}(\boldsymbol{H})-\frac{x}{2} h_{21}}{h_{11}}, & 0 \leq \xi \leq \mu_{2} \text { and } \\ & \eta_{4}(\xi) \leq x \leq L_{1}^{\max }(\xi)\end{cases}
$$

where $\mu_{2} \triangleq \frac{h_{12}}{h_{11}+h_{12}}$ and $\eta_{4}(\xi) \triangleq 2(1-\xi) h_{12}-2 \xi h_{11}$. In the following, we derive expressions for $L_{2}^{u p, \xi}(x)$ and $L_{2}^{\text {down, } \xi}(x)$ for scenario (b) $\left(h_{21} \leq h_{22}\right)$; and (c) $\left(h_{12} \leq h_{11}\right.$ and $h_{21}>$ $\left.h_{22}\right)$.

Similarly, for scenarios (b) and (c) also, by using the upward and downward extension methods we derive the expressions for $L_{2}^{u p, \xi}(x)$ and $L_{2}^{\text {down }, \xi}(x)$ respectively by constructing rectangles which lie inside $R_{/ /}(\boldsymbol{H})$ and have the maximum possible vertical lengths for a given horizontal length. It turns out that the expression for $\left(L_{2}^{u p, \xi}(x), L_{2}^{d o w n, \xi}(x)\right)$ is exactly the same as that for scenario (a).

\section{APPENDiX C \\ PROOF OF LEMMA 3}

Proof: To prove (29) we consider its R.H.S. $L_{2}^{1-\xi}(x)$. From (24) the R.H.S. is given by

$$
L_{2}^{(1-\xi)}(x)=2 \min \left(L_{2}^{u p,(1-\xi)}(x), L_{2}^{\text {down },(1-\xi)}(x)\right),
$$

where the expression for $L_{2}^{u p,(1-\xi)}(x)$ is given by,

Case I: For $0 \leq(1-\xi) \leq \frac{h_{11}}{h_{11}+h_{12}}$, i.e. $\frac{h_{12}}{h_{11}+h_{12}} \leq \xi \leq 1$ From (25) we have

$$
\begin{aligned}
& L_{2}^{u p,(1-\xi)}(x)=\left\{\frac{-(1-\xi) \operatorname{det}(\boldsymbol{H})-\frac{x}{2} h_{21}}{h_{11}}, \quad 0 \leq x \leq L_{1}^{\max }(1-\xi)\right. \\
& \stackrel{(a)}{=}\left\{\frac{-(1-\xi) \operatorname{det}(\boldsymbol{H})-\frac{x}{2} h_{21}}{h_{11}}, \quad 0 \leq x \leq L_{1}^{\max }(\xi)\right. \\
& \stackrel{(b)}{=} L_{2}^{\text {down, },}(x) \text {, }
\end{aligned}
$$

where step (a) follows from (22) and step (b) follows from (28).

Case II: For $\frac{h_{11}}{h_{11}+h_{12}} \leq(1-\xi) \leq 1$, i.e. $0 \leq \xi \leq \frac{h_{12}}{h_{11}+h_{12}}$, from (26) we have

$$
\begin{aligned}
L_{2}^{u p,(1-\xi)}(x)= & \begin{cases}\frac{-\xi \operatorname{det}(\boldsymbol{H})-\frac{x}{2} h_{22}}{h_{12}}, & 0 \leq x \leq \eta_{3}(1-\xi) \\
\frac{-(1-\xi) \operatorname{det}(\boldsymbol{H})-\frac{x}{2} h_{21}}{h_{11}}, & \eta_{3}(1-\xi) \leq x \leq L_{1}^{\max }(1-\xi)\end{cases} \\
& \stackrel{(a)}{=} \begin{cases}\frac{-\xi \operatorname{det}(\boldsymbol{H})-\frac{x}{2} h_{22}}{h_{12}}, & 0 \leq x \leq \eta_{4}(\xi) \\
\frac{-(1-\xi) \operatorname{det}(\boldsymbol{H})-\frac{x}{2} h_{21}}{h_{11}} & \eta_{4}(\xi) \leq x \leq L_{1}^{\max }(\xi)\end{cases} \\
& \stackrel{(b)}{=} L_{2}^{\text {down }, \xi}(x),
\end{aligned}
$$

where step (a) follows from two facts, firstly that $\eta_{3}(1-\xi) \triangleq$ $2(1-\xi) h_{12}-2 \xi h_{11}=\eta_{4}(\xi)$ and secondly from (22). Step 
(b) follows from (27). Therefore we have,

$$
L_{2}^{u p,(1-\xi)}(x)=L_{2}^{d o w n, \xi}(x), \quad \xi \in[0,1], x \in\left[0, L_{1}^{\max }(\xi)\right]
$$

From (102) we also have

$$
L_{2}^{u p, \xi}(x)=L_{2}^{\text {down },(1-\xi)}(x), \quad \xi \in[0,1], x \in\left[0, L_{1}^{\max }(\xi)\right]
$$

Using (102) and (103) in (99) we finally have

$$
\begin{aligned}
L_{2}^{(1-\xi)}(x) & =2 \min \left(L_{2}^{\text {up },(1-\xi)}(x), L_{2}^{\text {down },(1-\xi)}(x)\right) \\
& =2 \min \left(L_{2}^{\text {down }, \xi}(x), L_{2}^{\text {up }, \xi}(x)\right) \\
& \stackrel{(a)}{=} L_{2}^{\xi}(x) \\
& =\text { L.H.S. }
\end{aligned}
$$

where step (a) follows from (24). This therefore completes the proof.

\section{APPENDIX D}

\section{PROOF OF THEOREM 1}

To Prove this theorem we need the following Lemma.

Lemma 4. For a fixed $x \in\left[0, L_{1}^{\max }(1 / 2)\right]$, the function $L_{2}^{\xi}(x)$ attains its maximum at $\xi=1 / 2$, i.e.

$$
L_{2}^{\xi}(x) \leq L_{2}^{1 / 2}(x) \quad \forall \quad \xi \in[f(x), 1 / 2]
$$

where for any $0 \leq x \leq L_{1}^{\max }(1 / 2), f(x)$ is the unique ${ }^{16}$ value such that

$$
L_{1}^{\max }(f(x))=x \text { and } f(x) \leq 1 / 2 .
$$

Proof: To prove Lemma 4 we consider two cases (a) $h_{12} \leq h_{11}$; and (b) $h_{12} \geq h_{11}$. From Lemma 3 , we know that for a fixed $x \in\left[0, L_{1}^{\max }(1 / 2)\right], L_{2}^{\xi}(x)$ is symmetric about $\xi=1 / 2$, hence we consider $\xi$ only in the range $[0,1 / 2]$. The proof of Lemma 4 is as follows

Case(a) $h_{12} \leq h_{11}$ :

$$
h_{12} \leq h_{11}, \quad \text { i.e. } \quad 1 / 2 \leq \frac{h_{11}}{h_{11}+h_{12}} .
$$

Since $\frac{h_{11}}{h_{11}+h_{12}} \geq 1 / 2$ and $\xi \in[0,1 / 2]$, we have $\xi \leq \frac{h_{11}}{h_{11}+h_{12}}$. Therefore from (25) we have

$$
L_{2}^{u p, \xi}(x)=\frac{-\xi \operatorname{det}(\boldsymbol{H})-\frac{x}{2} h_{21}}{h_{11}}, 0 \leq x \leq L_{1}^{\max }(1 / 2) .
$$

From the above equation it is clear that $L_{2}^{u p, \xi}(x)$ is an increasing function of $\xi \in[0,1 / 2]$ and therefore for any $\xi \in[0,1 / 2]$ we have

$$
\xi \leq 1 / 2 \Longrightarrow L_{2}^{u p, \xi}(x) \leq L_{2}^{u p, 1 / 2}(x) .
$$

From (103) we know that $L_{2}^{u p, \xi}(x)=L_{2}^{\text {down, }(1-\xi)}(x)$, and therefore for $\xi=1 / 2$

$$
L_{2}^{u p, 1 / 2}(x)=L_{2}^{\text {down }, 1 / 2}(x) .
$$

\footnotetext{
${ }^{16}$ Uniqueness follows from the fact that $L_{1}^{\max }(\xi)$ is continuous, increases linearly when $\xi \in[0,1 / 2]$, has a unique maximum at $\xi=1 / 2$, and $L_{1}^{\max }(\xi)=L_{1}^{\max }(1-\xi)$.
}

using (110) in (24) we have

$$
L_{2}^{u p, 1 / 2}(x)=L_{2}^{\text {down }, 1 / 2}(x)=L_{2}^{\xi=1 / 2}(x) / 2
$$

and hence using this along with (109), for any fixed $x=$ $\left[0, L_{1}^{\max }(1 / 2)\right]$ we have

$$
\begin{array}{r}
L_{2}^{u p, \xi}(x) \leq L_{2}^{\xi=1 / 2}(x) / 2 \text {,i.e. } \\
2 \min \left(L_{2}^{u p, \xi}(x), L_{2}^{\text {down }, \xi}(x)\right) \leq L_{2}^{\xi=1 / 2}(x) \text {, i.e. } \\
L_{2}^{\xi}(x) \leq L_{2}^{\xi=1 / 2}(x) .
\end{array}
$$

Therefore for case(a) $\left(h_{12} \leq h_{11}\right)$ and $\xi \in[0,1 / 2]$ finally we have

$$
L_{2}^{\xi}(x) \leq L_{2}^{\xi=1 / 2}(x), \quad 0 \leq x \leq L_{1}^{\max }(1 / 2) .
$$

Case(b) $h_{11} \leq h_{12}$ :

$$
h_{11} \leq h_{12}, \quad \text { i.e. } \quad 1 / 2 \leq \frac{h_{12}}{h_{11}+h_{12}} .
$$

For this case, in order to prove $L_{2}^{\xi}(x) \leq L_{2}^{\xi=1 / 2}(x)$, we further consider two different cases on the basis of the values of $x \in$ $\left[0, L_{1}^{\max }(1 / 2)\right](\mathrm{b} . \mathrm{I}): x \in\left[0, h_{12}-h_{11}\right]$; and (b.II): $x \in\left[h_{12}-\right.$ $\left.h_{11}, L_{1}^{\max }(1 / 2)\right]$.

case (b.I): $x \in\left[0, h_{12}-h_{11}\right]$

From (27) we have

$$
\begin{aligned}
\eta_{4}(\xi) & =2(1-\xi) h_{12}-2 \xi h_{11} \\
& =2 h_{12}-2 \xi\left(h_{11}+h 12\right) .
\end{aligned}
$$

From the above equation it is cleat that $\eta_{4}(\xi)$ is monotonically decreasing with $0 \leq \xi \leq 1 / 2$ and therefore we have

$$
\begin{gathered}
\eta_{4}(1 / 2) \leq \eta_{4}(\xi) \leq \eta_{4}(0) \\
h_{12}-h_{11} \leq \eta_{4}(\xi) \leq 2 h_{12}
\end{gathered}
$$

and since we know that $x \in\left[0, h_{12}-h_{11}\right]$, hence for any value of $\xi \in[0,1 / 2], x$ will always be less than $\eta_{4}(\xi)$, i.e. $x \leq \eta_{4}(\xi)$. Therefore from (27) we have

$$
L_{2}^{\text {down }, \xi}(x)=\frac{-\xi \operatorname{det}(\boldsymbol{H})-\frac{x}{2} h_{22}}{h_{12}} .
$$

From the above equation it is clear that for a fixed $x \in\left[0, h_{12}-\right.$ $h_{11}$ ], $L_{2}^{\text {down, } \xi}(x)$ is a monotonically increasing function of $\xi \in[0,1 / 2]$. Therefore for any $\xi \in[0,1 / 2]$ we have

$$
\begin{array}{r}
L_{2}^{\text {down }, \xi}(x) \leq L_{2}^{\text {down }, 1 / 2}(x)=L_{2}^{\xi=1 / 2}(x) / 2, \quad \text { i.e. } \\
\min \left(L_{2}^{\text {up }, \xi}(x), L_{2}^{\text {down }, \xi}(x)\right) \leq L_{2}^{\xi=1 / 2}(x) / 2, \quad \text { i.e. } \\
2 \min \left(L_{2}^{\text {up }, \xi}(x), L_{2}^{\text {down }, \xi}(x)\right) \leq L_{2}^{\xi=1 / 2}(x), \text { i.e. } \\
L_{2}^{\xi}(x) \leq L_{2}^{\xi=1 / 2}(x) .
\end{array}
$$

Therefore for case(b.I) finally we have

$$
L_{2}^{\xi}(x) \leq L_{2}^{\xi=1 / 2}(x), \quad x \in\left[0, h_{12}-h_{11}\right] .
$$

case (b.II) $x \in\left[h_{12}-h_{11}, L_{1}^{\max }(1 / 2)\right]$ :

From (26) we have

$$
\begin{aligned}
\eta_{3}(\xi) & =2 \xi h_{12}-2(1-\xi) h_{11} \\
& =2 \xi\left(h_{12}+h_{11}\right)-2 h 11
\end{aligned}
$$


It is clear from the above equation that $\eta_{3}(\xi)$ is monotonically increasing with $\xi$ and hence for $\xi \in[0,1 / 2]$ we have

$$
\begin{aligned}
\eta_{3}(0) & \leq \eta_{3}(\xi) \leq \eta_{3}(1 / 2), \quad \text { i.e. } \\
-2 h_{11} & \leq \eta_{3}(\xi) \leq h_{12}-h_{11}
\end{aligned}
$$

Since $x \in\left[h_{12}-h_{11}, L_{1}^{\max }(1 / 2)\right]$ we have

$$
\eta_{3}(\xi) \leq h_{12}-h_{11} \leq x, \text { i.e. } \eta_{3}(\xi) \leq x .
$$

Therefore for case(b.II), from (25) and (26) we have

$$
L_{2}^{u p, \xi}(x)=\frac{-\xi \operatorname{det}(\boldsymbol{H})-\frac{x}{2} h_{21}}{h_{11}}, h_{12}-h_{11} \leq x \leq L_{1}^{\max }(1 / 2)
$$

It is clear that $L_{2}^{u p, \xi}(x)$ is monotonically increasing with $\xi$ and hence using the similar argument as for (112) we can show that

$$
L_{2}^{\xi}(x) \leq L_{2}^{\xi=1 / 2}(x) .
$$

Therefore using (119) and (123) ${ }^{17}$ for case (b) $\left(h_{12} \geq h_{11}\right)$ we have

$$
L_{2}^{\xi}(x) \leq L_{2}^{\xi=1 / 2}(x), 0 \leq x \leq L_{1}^{\max }(1 / 2) .
$$

Therefore finally from (124) and (112) and Lemma 3 we have for any $\xi \in[0,1]$

$$
L_{2}^{\xi}(x) \leq L_{2}^{\xi=1 / 2}(x) .
$$

This completes the proof of Lemma 4.

Proof: Next using this Lemma we prove Theorem 1.

Towards this end, we consider an arbitrary $\xi \in[0,1 / 2]$, for which we show that $R_{Z F}\left(\boldsymbol{H}, P_{0} / \sigma, \xi\right) \subseteq R_{Z F}\left(\boldsymbol{H}, P_{0} / \sigma, 1 / 2\right)$. For $\xi \in[1 / 2,1]$, the proof is similar due to the symmetricity of the $L_{2}^{\xi}(x)$ and $L_{1}^{\max }(\xi)$ functions (see Remark 1 and Lemma 3$)$. For a given $\xi \in[0,1 / 2]$ let $\left(R_{1}, R_{2}\right) \in$ $R_{Z F}\left(\boldsymbol{H}, P_{0} / \sigma, \xi\right)$. From (14), we know that there exists a $\operatorname{Rect}\left(L_{1} \geq 0, L_{2} \geq 0, D(\boldsymbol{H}, \xi)\right) \subset R_{/ /}(\boldsymbol{H})$ which corresponds to this rate pair $\left(R_{1}, R_{2}\right)$. Further from proposition 2 , it follows that there exists

$$
L_{2}^{\xi}\left(L_{1}\right) \geq L_{2}
$$

From Lemma (4) we know that for any a given $\xi \in[0,1 / 2]$ and $0 \leq L_{1} \leq L_{1}^{\max }(\xi)$, there exists

$$
L_{2}^{\xi=1 / 2}\left(L_{1}\right) \geq L_{2}^{\xi}\left(L_{1}\right)
$$

From (126) and (125) we get

$$
L_{2}^{\xi=1 / 2}\left(L_{1}\right) \geq L_{2} \text {. }
$$

We know that for $\xi=1 / 2$ there exists a rectangle $\operatorname{Rect}\left(L_{1}, L_{2}^{\xi=1 / 2}\left(L_{1}\right), D(\boldsymbol{H}, 1 / 2)\right) \quad \subset \quad R_{/ /}(\boldsymbol{H})$. From (127) it therefore follows that there will exists a rectangle $\operatorname{Rect}\left(L_{1}, L_{2}, D(\boldsymbol{H}, 1 / 2)\right) \quad \subset$ $\operatorname{Rect}\left(L_{1}, L_{2}^{\xi=1 / 2}\left(L_{1}\right), D(\boldsymbol{H}, 1 / 2)\right) \quad$ and hence $\operatorname{Rect}\left(L_{1}, L_{2}, D(\boldsymbol{H}, 1 / 2)\right) \quad \subset \quad R_{/ /}(\boldsymbol{H})$. The rate pair corresponding to the rectangle $\operatorname{Rect}\left(L_{1}, L_{2}, D(\boldsymbol{H}, 1 / 2)\right)$ is $\left(R_{1}, R_{2}\right)$ and therefore $\left(R_{1}, R_{2}\right) \in R_{Z F}\left(\boldsymbol{H}, P_{0} / \sigma, 1 / 2\right)$.

\footnotetext{
${ }^{17}$ In (119), $x \in\left[0, h_{12}-h_{11}\right]$, whereas in (123), $x \in\left[h_{12}-\right.$ $\left.h_{11}, L_{1}^{\max }(1 / 2)\right]$, both of these cases we have the same result and for the union of both of these cases $x \in\left[0, L_{1}^{\max }(1 / 2)\right]$
}

\section{APPENDIX E \\ PROOF OF THEOREM 2}

Proof: The proof of Theorem 2 is as follows. Let $(a, \alpha a)$ be any arbitrary rate pair of the form $(r, \alpha r)$ lying strictly inside the rate region $R_{Z F}\left(\boldsymbol{H}, P_{0} / \sigma, \xi\right)$ and which does not lie on the boundary $R_{Z F}^{B d}\left(\boldsymbol{H}, P_{0} / \sigma, \xi\right)$ (see the point $P$ in Fig. ??).

We then show that there exists the unique rate pair $\left(a^{\star}, \alpha a^{\star}\right)$ which lies on the boundary $R_{Z F}^{B d}\left(\boldsymbol{H}, P_{0} / \sigma, \xi\right)$ such that $a^{\star}>$ $a$. This shows that the rate pair $\left(R_{\max }^{\alpha}(\xi), \alpha R_{\max }^{\alpha}(\xi)\right)=$ $\left(a^{\star}, \alpha a^{\star}\right)$ is the unique rate pair of the form $(r, \alpha r)$ which lies on the boundary.

Since, the rate pair $(a, \alpha a) \in R_{Z F}\left(\boldsymbol{H}, P_{0} / \sigma, \xi\right)$, from (14) such a pair $(a, \alpha a)$ will correspond to some rectangle $\operatorname{Rect}(y, z>0, D(\boldsymbol{H}, \xi))$ inside the parallelogram $R_{/ /}(\boldsymbol{H})$, where $0 \leq y<L_{1}^{\max }(\xi)$ such that

$$
\begin{gathered}
a=C\left(y, P_{0} / \sigma\right), \text { and } \\
\alpha a=C\left(z, P_{0} / \sigma\right) .
\end{gathered}
$$

From (17), it follows that for the $y$ given in (128), (i.e., for rate of User 1 given in (128)) the largest possible rate to the second user is given by

$$
a_{1}=C\left(L_{2}^{\xi}(y), P_{0} / \sigma\right) .
$$

From (18), it follows that the rate pair $\left(a, a_{1}\right)=$ $\left(C\left(y, P_{0} / \sigma\right), C\left(L_{2}^{\xi}(y), P_{0} / \sigma\right)\right)$ lies on the boundary $R_{Z F}^{B d}\left(\boldsymbol{H}, P_{0} / \sigma, \xi\right)$. Since, $a_{1}$ is the largest possible rate of User 2 when rate of User 1 is equal to $a$ (see the point $Q$ in Fig. ??). it follows that

$$
\begin{gathered}
a_{1}>\alpha a \\
C\left(L_{2}^{\xi}(y), P_{0} / \sigma\right) \stackrel{(a)}{>} C\left(z, P_{0} / \sigma\right) \text {, i.e. } L_{2}^{\xi}(y) \stackrel{(b)}{>} z,
\end{gathered}
$$

where step (a) follows from (130) and (129). Step (b) follows from the fact that $C\left(x, P_{0} / \sigma\right)$ is a monotonically increasing function of its first arguments. From the above equation it follows that $z$ lies in the range of the function $L_{2}^{\xi}(x)$. It also follows from the continuity and monotonicity of $L_{2}^{\xi}(x)$ that there will exist a unique $0 \leq t \leq L_{1}^{\max }(\xi)$ such that

$$
z=L_{2}^{\xi}(t)
$$

From the last two equations it follows that

$$
L_{2}^{\xi}(t)<L_{2}^{\xi}(y)
$$

and hence since $L_{2}^{\xi}(x)$ is monotonically deceasing we have

$$
y<t
$$

$$
C\left(y, P_{0} / \sigma\right) \stackrel{(a)}{<} C\left(t, P_{0} / \sigma\right), \text { i.e. } a \stackrel{(b)}{<} C\left(t, P_{0} / \sigma\right),
$$

where step (a) follows from Result 2 and step (b) follows from (128). We have shown the rate pair $\left(C\left(t, P_{0} / \sigma\right), \alpha a\right)$ by the point $R$ in Fig. 12.

We now define the function

$$
f(x) \triangleq \alpha C\left(x, P_{0} / \sigma\right)-C\left(L_{2}^{\xi}(x), P_{0} / \sigma\right),
$$

where $x \in\left[0, L_{1}^{\max }(\xi)\right]$. With increasing $x, C\left(x, P_{0} / \sigma\right)$ increases (see Result 2) and $C\left(L_{2}^{\xi}(x), P_{0} / \sigma\right)$ decreases (as 


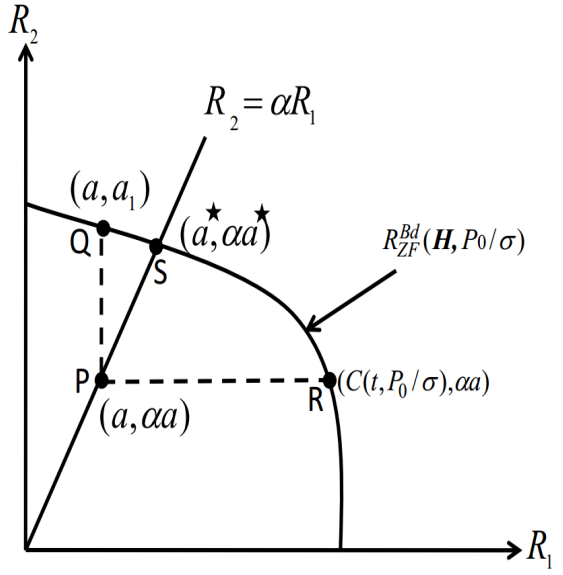

Fig. 12: A typical proposed rate region boundary.

$L_{2}^{\xi}(x)$ is a monotonically decreasing function of $x$, see Lemma 1). Hence $f(x)$ is a monotonically increasing function of $x$. Further, since $C\left(x, P_{0} / \sigma\right)$ and $L_{2}^{\xi}(x)$ are continuous functions, it follows that $f(x)$ is also continuous. It is clear that

$$
f(y) \stackrel{(a)}{=} \alpha C\left(y, P_{0} / \sigma\right)-C\left(L_{2}^{\xi}(y), P_{0} / \sigma\right) \stackrel{(b)}{=} \alpha a-a_{1} \stackrel{(c)}{<} 0,
$$

where step (a) follows from (135), step (b) follows from (128) and (130), and step (c) follows from (131). Similarly

$$
\begin{aligned}
f(t) & \stackrel{(a)}{=} \alpha C\left(t, P_{0} / \sigma\right)-C\left(L_{2}^{\xi}(t), P_{0} / \sigma\right) \\
& \stackrel{(b)}{=} \alpha C\left(t, P_{0} / \sigma\right)-C\left(z, P_{0} / \sigma\right) \\
& \stackrel{(c)}{=} \alpha C\left(t, P_{0} / \sigma\right)-\alpha a \\
& =\alpha\left(C\left(t, P_{0} / \sigma\right)-a\right) \stackrel{(d)}{>} 0
\end{aligned}
$$

where step (a) follows from (135), step (b) follows from the fact that $L_{2}^{\xi}(t)=z$ (see (132)). Step (c) follows from (129). Step (d) follows from (134).

Further, since $f(y)<0, y \in\left[0, L_{1}^{\max }(\xi)\right]$ and $f(x), x \in$ $\left[0, L_{1}^{\max }(\xi)\right]$ is monotonically increasing in $x$, it follows that $f(x=0)<0$. Similarly, $f\left(x=L_{1}^{\max }(\xi)\right)>0$ since $f(t)>0$ and $L_{1}^{\max }(\xi) \geq t$. Since, $f(x)$ is a monotonically increasing and continuous in $\left[0, L_{1}^{\max }(\xi)\right]$, and $f(0)<0, f(t)>0$, it follows that there exists a unique $x^{\star} \in\left[0, L_{1}^{\max }(\xi)\right]$ such that $f\left(x^{\star}\right)=0$ [19]. The uniqueness follows from the monotonicity of $f(x)$. That is, from (135) we have

$$
\alpha C\left(x^{\star}, P_{0} / \sigma\right)=C\left(L_{2}^{\xi}\left(x^{\star}\right), P_{0} / \sigma\right) .
$$

Let $a^{\star} \triangleq C\left(x^{\star}, P_{0} / \sigma\right)$ and therefore from (138) it follows that $\left.\alpha a^{\star}=C\left(L_{2}^{\xi}\left(x^{\star}\right), P_{0} / \sigma\right)\right)$. From (18), it is clear that the rate pair $\left(a^{\star}, \alpha a^{\star}\right) \in R_{Z F}^{B d}\left(\boldsymbol{H}, P_{0} / \sigma, \xi\right)$. Uniqueness of such a rate pair follows from the uniqueness of $x^{\star}$. Further since $f(y)<0=f\left(x^{\star}\right)$ (see Eq. 136) and $f(x)$ is monotonically increasing, it follows that

$$
x^{\star}>y \text {. }
$$

From Result (2) we know that $C\left(x, P_{0} / \sigma\right)$ is monotonically increasing in $x$ and therefore form (139) $a^{\star}=C\left(x^{\star}, P_{0} / \sigma\right)>$ $C\left(y, P_{0} / \sigma\right)=a$. Therefore we have shown that the unique rate pair $\left(a^{\star}, \alpha a^{\star}\right)$ lies on the boundary $R_{Z F}^{B d}\left(\boldsymbol{H}, P_{0} / \sigma, \xi\right)$ and $a^{\star}>a$ for any arbitrary choice of $a$, where $(a, \alpha a)$ lies strictly inside $R_{Z F}\left(\boldsymbol{H}, P_{0} / \sigma, \xi\right)$. As shown in Fig. ??, the point $\left(a^{\star}, \alpha a^{\star}\right)$ lies on the line $R_{2}=\alpha R_{1}$ and also on the boundary $R_{Z F}^{B d}\left(\boldsymbol{H}, P_{0} / \sigma, \xi\right)$. This therefore completes the proof.

\section{REFERENCES}

[1] H. Elgala, R. Mesleh, and H. Haas, "Indoor Optical Wireless Communication: Potential and State-of-the-Art," IEEE Communications Magazine, vol. 49, pp. 56-62, September 2011.

[2] S. Dimitrov and H. Hass, Principles of LED Light Communications: Towards Networked Li-Fi. Cambridge University Press, 2015.

[3] J. M. Kahn and J. R. Barry, "Wireless Infrared Communications," Proceedings of the IEEE, vol. 85, pp. 265-298, Feb 1997.

[4] J. Y. Wang, J. B. Wang, M. Chen, and X. Song, "Dimming scheme analysis for pulse amplitude modulated visible light communications," in 2013 International Conference on Wireless Communications and Signal Processing, pp. 1-6, Oct 2013.

[5] J. B. Wang, Q. S. Hu, J. Wang, M. Chen, and J. Y. Wang, "Tight bounds on channel capacity for dimmable visible light communications," Journal of Lightwave Technology, vol. 31, pp. 3771-3779, Dec 2013.

[6] A. Lapidoth, S. M. Moser, and M. A. Wigger, "On the Capacity of Free-Space Optical Intensity Channels," in 2008 IEEE International Symposium on Information Theory, pp. 2419-2423, July 2008.

[7] L. Wu, Z. Zhang, J. Dang, and H. Liu, "Capacity Lower Bounds of IM/DD AWGN Optical Wireless Channels Based on Fano's Inequality," in Wireless Communications Signal Processing (WCSP), 2015 International Conference on, pp. 1-5, Oct 2015.

[8] A. Thangaraj, G. Kramer, and G. Bcherer, "Capacity Upper Bounds for Discrete-time Amplitude-Constrained AWGN Channels," in 2015 IEEE International Symposium on Information Theory (ISIT), pp. 2321-2325, June 2015.

[9] J. G. Smith, "The Information Capacity of Amplitude and Variance Constrained Scalar Gaussian Channels," Information and Control, vol. 18, no. 3, p. 203 219, 1971.

[10] A. A. Farid and S. Hranilovic, "Capacity of Optical Intensity Channels with Peak and Average Power Constraints," in 2009 IEEE International Conference on Communications, pp. 1-5, June 2009.

[11] J. B. Wang, Q. S. Hu, J. Wang, M. Chen, Y. H. Huang, and J. Y. Wang, "Capacity Analysis for Dimmable Visible Light Communications," in 2014 IEEE International Conference on Communications (ICC), pp. 3331-3335, June 2014.

[12] T. Fath and H. Haas, "Performance Comparison of MIMO Techniques for Optical Wireless Communications in Indoor E nvironments," IEEE Transactions on Communications, vol. 61, pp. 733-742, Feb 2013.

[13] T. V. Pham, H. L. Minh, Z. Ghassemlooy, T. Hayashi, and A. T. Pham, "Sum-Rate Maximization of Multi-User MIMO Visible Light Communications," in 2015 IEEE International Conference on Communication Workshop (ICCW), pp. 1344-1349, June 2015.

[14] H. Shen, Y. Deng, W. Xu, and C. Zhao, "Rate-Maximized Zero-Forcing Beamforming for VLC Multiuser MISO Downlinks," IEEE Photonics Journal, vol. 8, pp. 1-13, Feb 2016.

[15] A. Chaaban, Z. Rezki, and M. S. Alouini, "On the Capacity of the 2-User IM-DD Optical Broadcast Channel," in 2015 IEEE Globecom Workshops (GC Wkshps), pp. 1-6, Dec 2015.

[16] A. Chaaban, Z. Rezki, and M. S. Alouini, "On the Capacity of the Intensity-Modulation Direct-Detection Optical Broadcast Channel," IEEE Transactions on Wireless Communications, vol. 15, pp. 31143130, May 2016.

[17] T. Komine and M. Nakagawa, "Fundamental Analysis for Visible-Light Communication System Using LED Lights," IEEE Transactions on Consumer Electronics, vol. 50, pp. 100-107, Feb 2004.

[18] B. Li, J. Wang, R. Zhang, H. Shen, C. Zhao, and L. Hanzo, "Multiuser MISO Transceiver Design for Indoor Downlink Visible Light Communication Under Per-LED Optical Power Constraints," IEEE Photonics Journal, vol. 7, pp. 1-15, Aug 2015.

[19] W. Rudin, Principles of Mathmatical Analysis. McGraw-Hill, 1976. 